\title{
Extremal Theory for Convex Matchings in Convex Geometric Graphs*
}

\author{
Y. S. Kupitz and M. A. Perles \\ Institute of Mathematics, The Hebrew University of Jerusalem, \\ Jerusalem, Israel \\ Communicated by Imre Bárány and János Fach
}

\begin{abstract}
A convex geometric graph $G$ of order $n$ consists of the set of vertices of a plane convex $n$-gon $P$ together with some edges and/or diagonals of $P$ as edges. Call $G l$-free if $G$ does not have $l$ disjoint edges in convex position.

We answer the following questions:

(a) What is the maximum possible number of edges of $G$ if $G$ is $l$-free (as a function of $n$ and $l$ )?

(b) What is the minimum possible number of edges of $G$ if $G$ is $l$-free and saturated, i.e., if $G \cup\{e\}$ is not $l$-free for any edge or diagonal $e$ of $P$ that is not already in $G$.

We also fully describe the graphs $G$ where the maximum (in (a)) or the minimum (in (b)) is attained. Then we remove the word "disjoint" from the definition of " $l$-free" and do the same over again. The results obtained are quite similar and closely related to the corresponding results (Turán's theorem, etc.) in extremal abstract graph theory.
\end{abstract}

\section{Introduction}

Before introducing the geometric objects mentioned in the title, we would like to illustrate, by means of a classical example from abstract graph theory, what we mean by "extremal theory."

Definition. In what follows we always assume that the graph $G$ is of order $n$. Call a graph $G q$-free ( $q \geq 2$ ) if it has no complete subgraph of order $q$. Call $G q$-saturated if $G$ is $q$-free, but is not included in any other $q$-free graph on the same set of vertices.

\footnotetext{
* Editors' note: This paper was accepted for the special issue of Discrete \& Computational Geometry (Volume 13, Numbers 3-4) devoted to the Lászlo Fejes Tóth Festschrift, but was not received in final form in time to appear in that issue.
} 
Define

$$
\begin{aligned}
\mathrm{T}_{q}(n) & =\max \{e(G): G \text { is a } q \text {-free graph (of order } n)\} \\
& =\max \{e(G): G \text { is a } q \text {-saturated graph\}, } \\
\mathrm{t}_{q}(n) & =\min \{e(G): G \text { is a } q \text {-saturated graph (of order } n)\}
\end{aligned}
$$

Call $G$ (of order $n$ ) $q$-extremal if $G$ is $q$-free and has $T_{q}(n)$ edges. Call $G q$-minimal if $G$ is $q$-saturated and has $\mathrm{t}_{q}(n)$ edges.

Results. (1) If $n=a(q-1)+r, 0 \leq r \leq q-1$, then

$$
\mathrm{T}_{q}(n)=\left(\begin{array}{l}
n \\
2
\end{array}\right)-(q-1)\left(\begin{array}{l}
a \\
2
\end{array}\right)-r a .
$$

Note that $\mathrm{T}_{2}(n)=0$. Another expression for $\mathrm{T}_{q}(n)$, that does not involve $a$ explicitly, is

$$
\mathrm{T}_{q}(n)=\frac{n^{2}}{2}\left(1-\frac{1}{q-1}\right)-\frac{r(q-1-r)}{2(q-1)},
$$

where, as before, $0 \leq r \leq q-1$ and $n \equiv r(\bmod (q-1))$. The "error term"

$$
r(q-1-r) / 2(q-1)
$$

is always between 0 and $(q-1) / 8$.

(2) $G$ is $q$-extremal iff $G$ is a complete $(q-1)$-partite graph with nearly equal parts (i.e., $G=\mathrm{K}_{a_{1}, a_{2}, \ldots . a_{q-1}}$, where $a_{i}=[(n+i-1) /(q-1)]$ for $\left.i=1,2, \ldots, q-1\right)$.

(3) $\mathrm{t}_{q}(n)= \begin{cases}\left(\begin{array}{l}n \\ 2\end{array}\right) & \text { for } n<q, \\ \left(\begin{array}{l}n \\ 2\end{array}\right)-\left(\begin{array}{c}n-q+2 \\ 2\end{array}\right) & \text { for } n \geq q .\end{cases}$

(4) $G$ is $q$-minimal iff:

(a) $G=\mathrm{K}_{n}$, when $n<q$.

(b) $G=\mathrm{K}_{n} \backslash \mathrm{K}_{n-q+2}$, i.e., $G$ is obtained from $\mathrm{K}_{n}$ by removing the edges of a complete subgraph of order $n-q+2$, when $n \geq q$.

Results (1) and (2) are due to Turán [T]. See p. 72 of [B] for an English presentation. Results (3) and (4) are due to Erdös et al. [EHM].

In the extremal theory presented in this paper we replace the abstract graph $G$ by a convex geometric graph (cgg) $G$. This is a graph whose vertices are points in the Euclidean plane $\mathbb{R}^{2}$. The set $V$ of vertices is assumed to be in convex position, i.e., either $\# V \leq 2$ or $V$ is the set of vertices of a convex polygon. The edges of $G$ are closed, nondegenerate line segments with endpoints in $V$.

Two edges of $G$ that have no vertex in common may cross, or they may be disjoint as line segments. This distinction between crossing and noncrossing vertex-disjoint edges makes much of the difference between abstract graph theory and geometric graph theory. 
The "forbidden subgraph" $\mathrm{K}_{q}$ of [T] and [EHM] is replaced by a variety of forbidden subconfigurations, where the main variants are $l$-matchings, convex $l$-matchings, and convex sets of $l$-edges (definitions follow).

Definition. Let $G=(V, E)$ be a cgg.

(a) An $l$-matching ( $l$-m for short) of $G$ is a set $\left\{e_{1}, \ldots, e_{l}\right\}$ of pairwise disjoint edges (disjoint as line segments, not just vertex-disjoint).

(b) A set $\left(e_{1}, \ldots, e_{l}\right)$ of edges of $G$ is convex if each $e_{i}$ is an edge of the convex polygon $\operatorname{conv}\left\{e_{1} \cup \cdots \cup e_{l}\right\}$ (or if $l \leq 1$ ). Note that two edges $e_{1}, e_{2}$ of $G$ form a convex set iff they do not cross.

The main body of the paper is devoted to the case where the forbidden configuration is a convex $l$-matching $(=l-\mathrm{cm})$. The other two cases $(l$-matching, not necessarily convex, and convex set of $l$-edges, not necessarily disjoint) are easier. They are treated briefly along with some other variants, in Sections 9 and 10.

As we shall see, there is a tight relationship between the extremal theory for abstract graphs (with respect to $\mathrm{K}_{l}$ ), and the extremal theory of convex $l$-matchings in cgg's. The resemblance becomes even closer when we replace convex $l$-matchings by convex sets of $l$ (not necessarily disjoint) edges.

We call a cgg $G l$-free if it includes no $l$-cm. $G$ is $l$-saturated if $G$ is $l$-free, but is not included in any other $l$-free cgg on the same set of vertices.

Define $\mathrm{cm}_{l}(n)$ (resp. $\mathrm{CM}_{l}(n)$ ) to be the minimal (resp. maximal) number of edges of an $l$-saturated cgg with $n$ vertices. $\mathrm{CM}_{l}(n)$ is, of course, also the maximal number of edges of an $l$-free $\operatorname{cgg}$ with $n$ vertices. Call a $\operatorname{cgg} G$ with $n$ vertices $l$-extremal if it is $l$-free and has $\mathrm{CM}_{l}(n)$ edges. Thus, $l$-saturated means $l$-free and maximal with respect to edge-set inclusion, whereas $l$-extreme means maximal with respect to the number of edges.

In Sections 2-6 we give a complete description of the $l$-saturated cgg's, calculate the number of edges $e(G)$ for each one of them, and determine the minimum $\mathrm{cm}_{l}(n)$. In Sections 7 and 8 we calculate $\mathrm{CM}_{l}(n)$ and determine the $l$-extreme cgg's. In Sections 9 and 10 we describe some extensions of the theory, and mention also some open problems for further research.

As we see in Section 8

$$
\mathrm{CM}_{l}(n)=\mathrm{T}_{l}(n)+n-l+1
$$

for $6 \leq 2 l \leq n$. In the extremal theory for convex sets of $l$ (not necessarily disjoint) edges, the corresponding extremal function turns out to be exactly $T_{l}(n)$ for $l \geq 3$ (see Section 9).

\section{Notation, Terminology, and Some Preparatory Results}

Let $V=\{v(0), \ldots, v(n-1)\}$ be a fixed finite set of $n$ points in $\mathbb{R}^{2}$. We assume $V$ to be in convex position, i.e., each point of $V$ is a vertex of the convex hull [ $V$ ] of $V$. If $n \geq 3$, we assume that the vertices $v(0), \ldots, v(n-1), v(0)$ appear in this cyclic order counterclockwise on the boundary bd[V] of $[V]$. We extend the indexing cyclically to all integers, so that $v(i)=v(j)$ iff $i \equiv j(\bmod n)\left(\operatorname{defining} v(k)==_{\text {def }} v(k-[k / n] n)\right.$ 
for all $k$ ). We denote by $\mathrm{CK}(n)$ the complete $\operatorname{cgg}$ on $V$, and regard all cgg's $G$ on $V$ as spanning subgraphs of $\mathrm{CK}(n)$.

The edges $[v(i), v(i+1)](0 \leq i<n)$ are the boundary edges of $\operatorname{CK}(n)$. The other edges of $\operatorname{CK}(n)$ are interior edges (or diagonals). An edge $e=[v(i), v(k)]$ of $\mathrm{CK}(n)$ divides $[V]$ into two closed subsets, called sides of $e$. If we think of $e$ as directed from $v(i)$ to $v(k)$, then we can identify them as the lefi side and the right side of $e$. We can always choose the labels $i, k$ so that $i<k<i+n$, and then precisely the vertices $v(i), v(i+1), \ldots, v(k-1), v(k)$ will be on the right side of $e$. If $e$ is a boundary edge, then the two sides of $e$ are $e$ and [V], respectively.

The length $\lambda(\vec{e})$ of a directed edge $\vec{e}$ of $\operatorname{CK}(n)$ is defined as the number of boundary edges on the right side of $\vec{e}$ (which is one less than the number of vertices on this side). Thus, if $i<k<i+n$, then $\lambda([v(i), v(k)])=k-i$ and $\lambda([v(k), v(i)])=$ $n-\lambda([v(i), v(k)])$.

Now consider the relative position of two undirected edges $e=[v(i), v(j)], f=$ $[v(h), v(k)]$ of $\mathrm{CK}(n)$. There are four possibilities: $e$ and $f$ may either coincide, share one vertex, be disjoint, or cross (i.e., have one common interior point). For three edges $e$, $f, g$ we need the notion of betweeness: $f$ lies between $e$ and $g$ if $e$ and $g$ lie on different (closed) sides of $f$. Note that under this definition $f$ lies between $f$ and $f$ !

We defined a set $E=\left\{e_{1}, \ldots, e_{l}\right\}$ of edges of $\mathrm{CK}(n)$ to be convex if each edge $e_{i}$ is a boundary edge of the convex hull $\left[e_{1} \cup \ldots \cup e_{l}\right]$. Thus $E$ is convex iff for each edge $e_{\mathrm{i}} \in E$ all edges of $E$ lie on one side of $e_{i}$. It follows that $E$ is convex iff:

(a) No two edges of $E$ cross.

(b) No edge of $E$ lies between two other edges of $E$.

We now turn our attention to a $\operatorname{cgg} G=(V, E)$. An edge $e$ of $G$ is extreme if it does not lie between two other edges of $G$, i.e., if (at least) one side of $e$ does not include any edge of $G$, except $e$.

Proposition 1.1. If $e$ is an edge of $G$, then on each side of $e$ there are extreme edges of $G$.

Proof. Assume $e=[v(i), v(k)]$, with $i<k<i+n$, and consider the right side of $e$. Among all edges of $G$ of the form $[v(\alpha), v(\beta)]$ with $i \leq \alpha<\beta \leq k$, choose one for which $\beta-\alpha$ is minimal. This is certainly an extreme edge of $G$. (It may coincide with $e$.)

Proposition 1.2. If $G$ has an $l$-cm, then it has an $l$-cm that consists of extreme edges only.

Proof. Suppose $L=\left\{e_{1}, \ldots, e_{l}\right\}$ is an $l-\mathrm{cm}$ in $G$. Direct each edge $e_{i}$ in such a way that $L$ lies on the left side of $e_{i}$ (if $l=1$, direct $e_{1}$ arbitrarily). Now replace each edge $e_{i}$ that is not extreme by an extreme edge $f_{i}$ on the right side of $e_{i}$.

We now introduce two operations on cgg's. For a $\operatorname{cgg} G=(V, E)$ define $\operatorname{Ext}(G)$ to be the cgg on $V$ whose edges are precisely the extreme edges of $G$, and let $\operatorname{Bet}(G)$ be the $\operatorname{cgg}$ on $V$ whose edges are all the edges of $\operatorname{CK}(n)$ that lie between two (not 
necessarily different) edges of $G$. Some useful properties of these operations are listed in the following proposition:

Proposition 1.3. Let $G=(V, E)$ be a cgg. Then:

(a) (i) $G \subset \operatorname{Bet}(G)$ and (ii) $\operatorname{ext}(G) \subset G$.

(b) If $G^{\prime}=\left(V, E^{\prime}\right)$ is another cgg on $V$, and $G \subset G^{\prime}$ (i.e., $E \subset E^{\prime}$ ), then $\operatorname{Bet}(G) \subset$ $\operatorname{Bet}\left(G^{\prime}\right)$.

(c) $\operatorname{Bet}(\operatorname{Bet}(G))=\operatorname{Bet}(G)$.

(d) $\operatorname{Ext}(\operatorname{Bet}(G))=\operatorname{Ext}(G)$.

(e) $\operatorname{Bet}(\operatorname{Ext}(G))=\operatorname{Bet}(G)$.

(f) $\operatorname{Ext}(\operatorname{Ext}(G))=\operatorname{Ext}(G)$.

Proof. The proofs are straightforward and we leave them to the reader. Some hints: For (c) and (d), use the fact that if $e$ and $f$ are edges of $G$, and if $f$ lies on the side $S$ of $e$, then $S$ includes one side of $f$. For (e) use Proposition 1.1.

Proposition 1.4. Let $G=(V, E)$ be a cgg. Then:

(a) If $G$ is l-free, then $\operatorname{Bet}(G)$ is also l-free.

(b) If $G$ is $l$-saturated, then $G=\operatorname{Bet}(G)=\operatorname{Bet}(\operatorname{Ext}(G))$.

Proof. For (a) use Propositions 1.2 and 1.3(a), (d). For (b) use (a) and Proposition 1.3(a)(i), (e).

Remark 1.5. Proposition 1.2 also holds for convex sets of edges that are not necessarily disjoint: If $C=\left\{e_{1}, \ldots, e_{l}\right\}$ is a convex set of $l$ edges of $G$, then there is a convex set $C^{\prime}=\left\{f_{1}, \ldots, f_{l}\right\}$ of $l$ extreme edges of $G$, and, in fact, $C^{\prime}$ uses at least as many vertices as $C$. It follows that Proposition 1.4(b) also holds for cgg's $G$ that are saturated (= inclusion maximal) with respect to the property of not having a convex set of $l$ edges (or a convex set of $l$ edges that use at least $l+c$ vertices, where $c$ is some prescribed integer $\geq 0$ ).

Proposition 1.6. A cgg $G^{*}=\left(V, E^{*}\right)$ is $\operatorname{Ext}(G)$ for some $l$-saturated cgg $G$ iff:

(i) $\operatorname{Ext}\left(G^{*}\right)=G^{*}$ (i.e., there is no edge of $E^{*}$ lying between two other edges).

(ii) $E^{*}$ does not contain an $l-\mathrm{cm}$.

(iii) If $\bar{e} \in E(\mathrm{CK}(n)) \backslash E\left(\operatorname{Bet}\left(G^{*}\right)\right)$, then $E^{*} \cup\{\bar{e}]$ does contain an l-matching.

Proof. Part (i) is true for the "Ext" of any cgg (see Proposition 1.3(f)). The rest follows from Propositions 1.2 and 1.4.

\section{Construction of $l$-Saturated cgg's-Type I}

Preliminary Remarks 2.1. If $l=1$, then a $\operatorname{cgg}(V, E)$ is $l$-free iff $E=\emptyset$, i.e., $\mathrm{cm}_{1}(n)=\mathrm{CM}_{1}(n)=0$. Assume therefore that $l \geq 2$. If \#V=n<2l, then $\mathrm{CK}(n)$ is $l$ free, and therefore $\mathrm{CK}(n)$ is the only $l$-saturated $\operatorname{cgg}$ on $V$, and $\mathrm{cm}_{l}(n)=\mathrm{CM}_{l}(n)=\left(\begin{array}{l}n \\ 2\end{array}\right)$. 
Assume therefore that $n \geq 2 l$. If $\# V=n=2 l$, then $\mathrm{CK}(n)$ has precisely two $l$-cm's. They are edge-disjoint, and together form the set of boundary edges of the convex $2 l$ gon $[V]$. Thus $G=(V, E)$ is $l$-saturated iff it misses one edge of each $l-\mathrm{cm}$. Thus $\mathrm{cm}_{l}(2 l)=\mathrm{CM}_{l}(2 l)=\left(\begin{array}{c}2 l \\ 2\end{array}\right)-2$.

We now describe a construction of $l$-saturated cgg's with $n$ vertices for $n \geq 2 l \geq 6$. These are called cgg's of type I. (In the next section we construct another kind of $l$ saturated cgg's, of type $\Pi$, for $n \geq 2 l \geq 4$.)

Put $m=l-1$ and choose a subset $A=\left\{a_{1}, \ldots, a_{m}\right\}$ of $V$. Assume, without loss of generality, that $a_{i}=v\left(\alpha_{i}\right)$ with $\alpha_{1}<\alpha_{2}<\cdots<\alpha_{m}<\alpha_{1}+n$, set $\alpha_{m+1}=\alpha_{1}+n$, and define, for $i=1, \ldots, m$,

$$
X_{i}=\left\{v(j): \alpha_{i}<j<\alpha_{i+1}\right\}, \quad x_{i}=\# X_{i}=\alpha_{i+1}-\alpha_{i}-1 .
$$

Thus the points $a_{1}, \ldots, a_{m}, a_{1}$ appear in this cyclic order on the boundary of [V], and $X_{i}$ is the set of vertices of [V] that lie strictly between $a_{i}$ and $a_{i+1}$ (or $a_{m}$ and $a_{1}$, for $i=m)$. We impose two conditions:

$x_{i}>0$ for all $i$, i.e., no two of the points $a_{i}$ are adjacent vertices of [V].

At least two of the $x_{i}$ 's are $\geq 2$, i.e., the points $a_{i}$ do not form one long succession of alternate vertices of $[V]$.

Remark. Condition (2.3) implies, of course, that $m \geq 2$, i.e., $l \geq 3$ (hence $n \geq 2 l \geq 6$ ).

Define $G^{*}=\left(V, E^{*}\right)$, where $E^{*}=\bigcup_{i=1}^{m}\left\{\left[v\left(\alpha_{i}-1\right), v\left(\alpha_{i}\right)\right],\left[v\left(\alpha_{i}\right), v\left(\alpha_{i}+1\right)\right]\right\}$. $E^{*}$ is the set of boundary edges of $\mathrm{CK}(n)$ that are incident with $A$. Condition (2.2) implies that $\# E^{*}=2 m=2(l-1)$, and that the edges of $E^{*}$ form a system of disjoint paths, each one of even length, on the boundary bd[V] of [V]. Condition (2.3) says that this system consists of at least two paths. Clearly, $G^{*}=\operatorname{Ext}\left(G^{*}\right)$. Finally define $G=(V, E)=\operatorname{Bet}\left(G^{*}\right)$. Now make the following observations:

\section{Proposition 2.2.}

(a) Ext $G=G^{*}$.

(b) $G$ is l-free.

(c) An edge e of $\mathrm{CK}(n)$ belongs to $G$ iff each side of e meets $A$.

(d) A vertex $v$ of $G$ is universal (i.e., $(n-1)$-valent) iff $v$ is an interior vertex of one of the paths that form the components of $E^{*}$. In particular, all points of $A$ are universal vertices of $G$.

(e) An edge of $\mathrm{CK}(n)$ is not an edge of $G$ iff it connects two vertices of the same set $X_{i}(1 \leq i \leq m)$. Hence $X_{i}$ is an independent set in $G$.

(f) $G$ is l-saturated.

(g) $\sum_{i=1}^{m} x_{i}=n-l+1$, and

$$
\begin{aligned}
e(G) & =\left(\begin{array}{l}
n \\
2
\end{array}\right)-\sum_{i=1}^{m}\left(\begin{array}{c}
x_{i} \\
2
\end{array}\right)=\left(\begin{array}{l}
n \\
2
\end{array}\right)+\frac{1}{2}(n-l+1)-\frac{1}{2} \sum_{i=1}^{m} x_{i}^{2} \\
& =\frac{1}{2}\left(n^{2}-l+1-\sum_{i=1}^{m} x_{i}^{2}\right) .
\end{aligned}
$$


Proof. (a) Follows from Proposition 1.3(d), with $\operatorname{Ext}\left(G^{*}\right)=G^{*}$.

(b) Follows from Proposition 1.4(a), since $G=\operatorname{Bet}\left(G^{*}\right)$, and $G^{*}$ is obviously $l$-free $\left(G^{*}\right.$ has no $l$-matching even as an abstract graph).

(c) $e \in E$ iff each side of $e$ includes an edge of $E^{*}$, and this happens iff each side of e meets $A$.

(d) Obvious.

(e) Follows from (c).

(f) Define, for $1 \leq k \leq m, e_{k}^{-}=\left[v\left(\alpha_{k}-1\right), v\left(\alpha_{k}\right)\right], e_{k}^{+}=\left[v\left(\alpha_{k}\right), v\left(\alpha_{k+1}\right)\right]$. (These are the two edges of $G^{*}$ incident with $a_{k}$.) Extend the indexing of $a_{k}, e_{k}^{-}, e_{k}^{+}$cyclically modulo $m$ to all integers, so that, e.g., $a_{k}=a_{l}$ iff $k \equiv l(\bmod m)$. Now add to $G$ an edge $e^{*}$ not in $E$. By (e), both endpoints of $e^{*}$ belong to the same set $X_{i}(1 \leq i \leq m)$. By (2.3) there is another set $X_{j}$ with $\# X_{j}=x_{j} \geq 2$. Choose the indexing in such a way that $i<j<i+m$. Note that $X_{i}$ lies between $a_{i}$ and $a_{i+1}$. Finally define

$$
L=\left\{e^{*}\right\} \cup\left\{e_{\nu}^{+}: i+1 \leq \nu \leq j\right\} \cup\left\{e_{v}^{-}: j+1 \leq v \leq i+m\right\} .
$$

(Here $e_{i+m}^{-}=e_{i}^{-}$. We choose from each pair $e_{\nu}^{-}, e_{\nu}^{+}$the edge that points away from $e^{*}$ and toward $X_{j}$.) Clearly, $L$ is an $l$-cm in $G \cup\left\{e^{*}\right\}$ (note that $e_{j}^{+}$and $e_{j+1}^{-}$are disjoint since $\# X_{j} \geq 2$ ). Thus $G$ is $l$-saturated.

$(\mathrm{g})$

$$
\sum_{i=1}^{m} x_{i}=\sum_{i=1}^{m} \# X_{i}=\#\left(\bigcup_{i=1}^{m} X_{i}\right)=n-\# A=n-l+1
$$

follows from the definition of the sets $X_{i}$. The rest follows from (e).

From Proposition 2.2(g) we infer:

Proposition 2.3. The maximum of $e(G)$ for the l-saturated cgg's $G$ of type $\mathrm{I}$, constructed here, is attained when and only when the numbers $x_{1}, \ldots, x_{m}$ are as equal as possible. If $n=(l-1)(q+1)+r, 1 \leq r \leq l-1(=m)$, that is, $n-l+1=(l-1) q+r$, choose $r$ times $x_{i}=q+1$, and $m-r$ times $x_{i}=q$ to obtain

$$
\begin{aligned}
& \max \{e(G): G \text { is an l-saturated cgg of type } \mathrm{I}, \boldsymbol{n} \text { vertices }\} \\
&=\left(\begin{array}{l}
n \\
2
\end{array}\right)-(l-1)\left(\begin{array}{l}
q \\
2
\end{array}\right)-r q .
\end{aligned}
$$

Proof. The first statement follows easily from Proposition 2.2(g). It remains only to check the calculation: the formula for $e(G)$ in Proposition 2.2(g) gives, for the minimizing sequence ( $r$ times $q+1$ and $m-r$ times $q$ ),

$$
\left(\begin{array}{l}
n \\
2
\end{array}\right)-\sum_{i=1}^{m}\left(\begin{array}{l}
x_{i} \\
2
\end{array}\right)=\left(\begin{array}{l}
n \\
2
\end{array}\right)-r\left(\begin{array}{c}
q+1 \\
2
\end{array}\right)-(m-r)\left(\begin{array}{l}
q \\
2
\end{array}\right),
$$

which is equal to the right-hand side of (2.4). Note that (2.4) also holds for $r=0$, i.e., for $n=(l-1)(q+1)(=(l-1) q+l-1)$. 
Note that, in view of $(0.1)$, the right-hand side of (2.4) is equal to $\mathrm{T}_{l}(n)+n-l+1$. (Please check.) As we see later (in Section 7), this is the maximum of $e(G)$ for $l$-free cgg's with $n$ vertices (i.e., this is $\mathrm{CM}_{l}(n)$ ). Section 7 does not depend on anything from Sections 3-6.

Proposition 2.4. The minimum of $e(G)$ for $l$-saturated cgg's of type I with $n$ vertices is obtained when, say, $x_{1}=\cdots=x_{m-2}=1, x_{m-1}=2$, and $x_{m}=n-2 l+2$, and is equal to $2(l-1) n-\left(2 l^{2}-3 l+2\right)$.

Proof. This follows by a simple calculation from Proposition 2.2(g).

\section{Construction of $l$-Saturated cgg's-Type II}

Fix a positive integer $d(=$ the jump number), put $m=(d+1) l-1, l \geq 2$, assume $n \geq m$, and choose a subset $A=\left\{a_{1}, \ldots, a_{m}\right\}$ of $V$ of size $m$. (No extra conditions this time, see (2.2) and (2.3).) As in Section 2 assume, without loss of generality that $a_{i}=v\left(\alpha_{i}\right)$, with $\alpha_{1}<\alpha_{2}<\cdots<\alpha_{m}<\alpha_{1}+n$, and define the sets $X_{i}$ and the numbers $x_{i}, 1 \leq i \leq m$, exactly as in (2.1). Extend the indexing of $a_{i}, X_{i}$, and $x_{i}$ cyclically modulo $m$ to all integers, and define $G^{*}=\left(V, E^{*}\right)$, where, this time, $E^{*}=\left\{\left[a_{i}, a_{i+d}\right]: 1 \leq i \leq m\right\}$.

$E^{*}$ is the set of edges on $A$ that have $d+1$ points of $A$ on one (closed) side, and $m-d+1$ points of $A$ on the other (closed) side. (From $m=(d+1) l-1 \geq 2 d+1$ it follows that $d+1<m-d+1$.) It can easily be checked that $\operatorname{Ext}\left(G^{*}\right)=G^{*}$.

Finally define $G=(V, E)=\operatorname{Bet}\left(G^{*}\right)$. In the following proposition we list the main properties of $G$ (compare Proposition 2.2).

\section{Proposition 3.1.}

(a) $\operatorname{Ext}(G)=G^{*}$.

(b) An edge e of $\mathrm{CK}(n)$ belongs to $G$ iff each (closed) side of e contains at least $d+1$ points of $A$.

(c) $G$ is l-free.

(d) $G$ is l-saturated.

(e) If $a_{i}, a_{j} \in A,|i-j| \leq \frac{1}{2} m$, then $\left[a_{i}, a_{j}\right]$ is not an edge of $G$ iff $|i-j|<d$.

(f) If $a_{i} \in A$ and $x \in X_{j}, i-m / 2 \leq j \leq i+m / 2$, then $\left[a_{i}, x\right]$ is not an edge of $G$ iff $i-d \leq j \leq i+d-1$.

(g) If $x \in X_{i}, y \in X_{j},|i-j| \leq \frac{1}{2} m$, then $[x, y]$ is not an edge of $G$ iff $|i-j| \leq d$.

(h) $\sum_{i=1}^{m} x_{i}=\#\left(\bigcup_{i=1}^{m} X_{i}\right)=\#(V \backslash A)=n-m$, and

$$
\begin{aligned}
e(G)= & \left(\begin{array}{l}
n \\
2
\end{array}\right)-m(d-1)-2 d(n-m)-\sum_{i=1}^{m}\left(\begin{array}{c}
x_{i} \\
2
\end{array}\right) \\
& -\sum\left\{x_{i} x_{j}: 1 \leq i<j \leq m \text { and }(j-i \leq d \text { or } j-i \geq m-d)\right\}
\end{aligned}
$$




$$
\begin{aligned}
= & \left(\begin{array}{l}
n \\
2
\end{array}\right)-m(d-1)-2 d(n-m)+\frac{1}{2}(n-m) \\
& -\frac{1}{2} \sum\left\{x_{i} x_{j}: 1 \leq i \leq m, 1 \leq j \leq m, \text { and }(|i-j| \leq d \text { or }|i-j| \geq m-d)\right\} .
\end{aligned}
$$

Proof. (a) Same as Proposition 2.2(a).

(b) Follows from $G=\operatorname{Bet}\left(G^{*}\right)$, and from the properties of $E^{*}$ listed before.

(c) By Proposition 1.4(a), it suffices to show that $G^{*}$ is $l$-free. Assume, on the contrary, that $G^{*}$ is not $l$-free, and let $L=\left\{e_{1}, \ldots, e_{l}\right\}$ be an $l$-cm in $G^{*}$. For $1 \leq i \leq l$, denote by $S_{i}$ the side of $e_{i}$ that does not meet any other edge of $L .\left(S_{i}\right.$ is well defined since $l \geq 2$.) By the remark preceding Proposition 3.1, each side $S_{i}$ contains at least $d+1$ points of $A$. However, the sides $S_{1}, \ldots, S_{l}$ are pairwise disjoint. ( $S_{i}$ is defined as the intersection of $[V]$ with one of the closed half-planes bounded by the line aff $e_{i}$.) This is impossible, since $\# A=m=(d+1) l-1<(d+1) l$.

(d) Let $e^{*}$ be an edge of $\mathrm{CK}(n)$ not in $G$. We must show that $G \cup\left\{e^{*}\right\}$ has an $l$ $\mathrm{cm}$. We will show, indeed, that $G^{*} \cup\left\{e^{*}\right\}$ has an $l$-cm. Let $S^{+}, S^{-}$be the two closed sides of $e^{*}$. By (b), one of those sides, say $S^{-}$, contains fewer than $d+1$ points of $A$. It follows that $S^{+} \backslash e^{*}$ contains at least $m-d=(d+1)(l-1)$ points of $A$. Assume $A \cap\left(S^{+} \backslash e^{*}\right)=\left\{a_{i}, a_{i+1}, \ldots, a_{i+t}\right\}$ where $m-d-1 \leq t<m$. Now define, for $1 \leq v \leq l-1, e_{v}=\left[a_{i+(\nu-1)(d+1)}, a_{i+v(d+1)-1}\right]$, and put $L=\left\{e_{1}, e_{2}, \ldots, e_{l-1}, e^{*}\right\}$. Then $L$ is an $l$-cm in $G^{*} \cup\left\{e^{*}\right\}$.

(e), (f) and (g) follow directly from (b). (Remember that $X_{i}$ is the set of points of $V$ that lie strictly between $a_{i}$ and $a_{i+1}$ on bd[V].)

(h) By (e), each point $a \in A$ is connected by nonedges in $G$ (i.e., by edges of $\mathrm{CK}(n)$ that are not in $G)$ to $2(d-1)$ other points of $A$. This takes account of the term $-m(d-1)$ in the formula. By ( $f$ ), each point $x \in X_{i}$ is connected by nonedges in $G$ to exactly $2 d$ points of $A$. This takes care of the term $-2 d(n-m)$. The remaining terms account for the nonedges that connect points of the same set $X_{i}$, or of two distinct sets $X_{i}, X_{j}$ $(\operatorname{see}(\mathrm{g}))$.

\section{Proposition 3.2.}

(a) Given $l$, $n$, and $d$, with $l \geq 3, d \geq 1$, and $n \geq(d+1) l-1(=m)$, all $l$-saturated cgg's $G$ of type II constructed here satisfy

$$
e(G) \geq\left(\begin{array}{l}
n \\
2
\end{array}\right)-m(d-1)-2 d(n-m)+\frac{1}{2}(n-m)-\frac{1}{2}(n-m)^{2} .
$$

Equality holds iff there is a chain of $d+1$ consecutive sets $X_{i}$ that contains all nonempty $X_{i}$ 's, i.e., iff, for some $i, \sum_{v=0}^{d} x_{i+v}=n-m$.

(b) If $l=2$, then we always obtain $e(G)=n$.

Proof. (a) In Proposition 3.1(h) we have

$$
\begin{aligned}
& \sum\left\{x_{i} x_{j}: 1 \leq i \leq m, 1 \leq j \leq m,(|i-j| \leq d \text { or }|i-j| \geq m-d)\right\} \\
& \leq \sum\left\{x_{i} x_{j}: 1 \leq i \leq m, 1 \leq j \leq m\right\}=\left(\sum_{i=1}^{m} x_{i}\right)^{2}=(n-m)^{2} .
\end{aligned}
$$




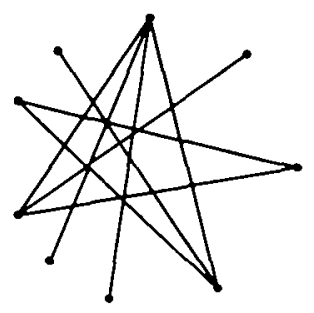

Fig. 1

Define $P:=\left\{i: 1 \leq i \leq m, x_{i}>0\right\}$. We consider $P$ as a subset of $\mathbb{Z}_{m}(=\mathbb{Z} / m \mathbb{Z})$. Equality holds in (3.2) iff the circular distance in $\mathbb{Z}_{m}$ between any two points of $P$ is $\leq d$. Since $m=(d+1) l-1 \geq 3 d+2$ (here we invoke the assumption $l \geq 3$ ), this implies that $P$ lies on an arc of length $d\left(=d+1\right.$ consecutive points) on the circuit $\mathbb{Z}_{m}$.

(b) If $l=2$, then $m=2 d+1$, and the condition " $|i-j| \leq d$ or $|i-j| \geq m-d "$ is satisfied by all $1 \leq i, j \leq m$. Thus the right-hand side of the formula for $e(G)$ in Proposition 3.1(h) reduces to $\left(\begin{array}{l}n \\ 2\end{array}\right)-m(d-1)-2 d(n-m)+\frac{1}{2}(n-m)-\frac{1}{2}(n-$ $m)^{2}$, which is equal to $n$ (please check). (For a simpler argument, see Remark 3.3(ii) below.)

Remark 3.3. (i) It follows from the definitions and from Propositions 2.2(a) and 3.1 (a) that types I and II are mutually disjoint, i.e., a cgg cannot be both of type I and of type II.

(ii) It is instructive to have a better look at case $l=2$ of the $l$-saturated $\mathrm{cgg}$ 's $G$ of

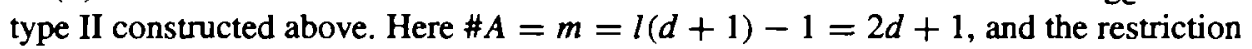
of $G$ to $A$ is a self-intersecting $(2 d+1)$-circuit (an "asterisk"). The vertices of $X_{i}$, that lie between $a_{i}$ and $a_{i+1}$ on bd [V], are connected to the common neighbor of $a_{i}$ and $a_{i+1}$, i.e., to the opposite vertex $a_{i-d}=a_{i+1+d}$ (see Fig. 1). Geometric graphs of this type, without the restriction of convexity, are the extremal 2-matching-free geometric graphs of order $n$. (See Remark 5.7 below and [E], [W], and [K].)

\section{Characterization of $l$-Saturated cgg's}

In this section and the next we show that the only $l$-saturated cgg's are those constructed in Section 2 (type I) and in Section 3 (type II).

Let $G=(V, E)$ be an $l$-saturated cgg, $\# V=n \geq 2 l \geq 4$. Define $G^{*}=\left(V, E^{*}\right)=$ $\operatorname{Ext}(G)$, and put $\mu=\# E^{*}$. Note that $\mu \geq 2$. (If $\mu=0$, then $E=E^{*}=\emptyset$, and $G$ is not $l$-saturated for any $l \geq 1$. If $\mu=1$, then $E=E^{*}=\{e\}$, and $G$ is not even 2-saturated, since we can add to $G$ another edge that meets $e$.) In fact, it will finally turn out that $\mu \geq 3$. By Proposition 1.4(b), $G=\operatorname{Bet}\left(G^{*}\right)$; thus it suffices to show that $E^{*}$ coincides with one of the sets $E^{*}$ defined in Sections 2 and 3.

Direct each edge $e$ of $E^{*}$ in such a way that no edge of $G$ (except $e$ ) lies on the right side of $e$. This can be done, since $e$ is an extreme edge of $G$. In case both sides of $e$ do not include any other edge of $G$, fix the direction of $e$ arbitrarily. (Later we see that this case never occurs.) Assume $E^{*}=\left\{e_{1}, \ldots, e_{\mu}\right\}$ and $e_{i}=\left[a_{i}, b_{i}\right], i=1,2, \ldots, \mu$. ( $e_{i}$ is directed from $a_{i}$ to $b_{i}$.) 
Of two directed edges in $\mathrm{CK}(n)$ with the same starting point or with the same endpoint, one must necessarily lie on the right side of the other. It follows that the $\mu$ vertices $a_{1}, \ldots, a_{\mu}$ are all different. The same for $b_{1}, \ldots, b_{\mu}$. We can find numbers $\alpha_{i}, 0 \leq \alpha_{i}<$ $n$, and $\beta_{i}$,

$$
\alpha_{i}<\beta_{i}<\alpha_{i}+n,
$$

such that $a_{i}=v\left(\alpha_{i}\right), b_{i}=v\left(\beta_{i}\right)(i=1,2, \ldots, \mu)$. Assume that $E^{*}$ has been indexed in such a way that $0 \leq \alpha_{1}<\alpha_{2}<\cdots<\alpha_{\mu}<n$. As we have done before, we extend the indexing of $e_{i}, a_{i}, b_{i}, \alpha_{i}$, and $\beta_{i}$ cyclically modulo $\mu$ to all integers $i$.

Proposition 4.1. $\beta_{1}<\beta_{2}<\cdots<\beta_{\mu}<\beta_{1}+n$.

Proof. We already know that the numbers $\beta_{1}, \ldots, \beta_{\mu}$ are all noncongruent modulo $n$. If $1 \leq i<j \leq \mu$ and $\beta_{i}>\beta_{j}$, then $\alpha_{i}<\alpha_{j}<\beta_{j}<\beta_{i}$ and therefore $e_{j}$ lies on the right side of $e_{i}$, contrary to our assumption. Thus $\beta_{1}<\beta_{2}<\cdots<\beta_{\mu}$. It remains to show that

$$
\beta_{\mu}<\beta_{1}+n
$$

If (4.2) fails, then (by (4.1)) $\beta_{1}+\eta<\beta_{\mu}<\alpha_{\mu}+n$ and therefore $\beta_{1}<\alpha_{\mu}$. However, then $e_{1}$ lies on the right side of $e_{\mu}$ (please check!), a contradiction.

For later reference, we list again all the inequalities conceming the indices $\alpha_{i}, \beta_{i}$ :

$$
\left\{\begin{array}{l}
0 \leq \alpha_{1}<\cdots<\alpha_{\mu}<n, \\
\beta_{1}<\beta_{2} \cdots<\beta_{\mu}<\beta_{1}+n, \\
\alpha_{i}<\beta_{i}<\alpha_{i}+n \quad(i=1,2, \ldots, \mu) .
\end{array}\right.
$$

Define $A=\left\{a_{1}, \ldots, a_{\mu}\right\}(\subset V), B=\left\{b_{1}, \ldots, b_{\mu}\right\}(\subset V)$. Proposition 4.1 implies that the correspondence $a_{i} \rightarrow b_{i}(i=1, \ldots, \mu)$ is a 1-1 orientation-preserving mapping of $A$ onto $B$. Now distinguish two cases:

Case I: $A=B$. We show that in this case $G$ is of type II.

Assume $b_{1}=a_{1+d}$, where $1 \leq d<\mu$ (note that $a_{1}=a_{1+\mu}$ and $b_{1} \neq a_{1}$, hence $1 \leq d \leq \mu$ implies $d<\mu$ ). Since $a_{i} \rightarrow b_{i}$ is an orientation-preserving bijection of $A$, it follows that $b_{i}=a_{i+d}$ for $i=1,2, \ldots, \mu$. Our next aim is to show that $\mu=(d+1) l-1$. This will clearly imply that our $\operatorname{cgg} G$ is one of the $l$-saturated cgg's of type II constructed at the beginning of Section 3, with $m=\mu$.

If $\mu \geq(d+1) l$, then $G^{*}$ (and therefore $G$ ) has an $l$-cm $L$ : Take

$$
L=\left\{e_{1+v(d+1)}: \nu=0,1,2, \ldots, l-1\right\} .
$$

(The vertices $a_{1}, b_{1}\left(=a_{d+1}\right), a_{d+2}, b_{d+2}\left(=a_{2 d+2}\right), a_{2 d+3}, b_{2 d+3}, \ldots, a_{1+(l-1)(d+1)}$, $b_{1+(l-1)(d+1)}\left(=a_{l(d+1)}\right)$ are all different and appear in this order on the boundary of [V].) Thus $\mu \leq l(d+1)-1$.

Now we use our assumption that $G$ is $l$-saturated to show that $\mu \geq l(d+1)-1$. Assume first $d>1$. Define $\bar{e}=\left[a_{1}, a_{d}\right]$ (directed from $a_{1}$ to $a_{d}$ ), $\bar{E}=\bar{E} \cup\{\bar{e}\}, \bar{G}=(V, \bar{E})$, and $\bar{G}^{*}=\operatorname{Ext}(\bar{G})=\left(V, \bar{E}^{*}\right)$. Note taht $\bar{e} \notin E$ and $\bar{E}^{*} \subset E^{*} \cup\{\bar{e}\}$ (check!). Since $G$ 
is $l$-saturated, $\bar{G}$ must include an $l$-cm. However, then $\bar{G}^{*}$ must also include an $l$-cm $L$ (see Proposition 1.2). Since $\bar{E}^{*} \subset E^{*} \cup\{\bar{e}\}$, and $G^{*}$ is $l$-free, $L$ consists of $\bar{e}$ and $l-1$ edges of $E^{*}$. The right side of $\bar{e}$ contains exactly $d$ points $a_{1}, \ldots, a_{d}$ of $A$. The right side of any edge in $E^{*}$ contains exactly $d+1$ points of $A$. Since the right sides of all edges in $L$ must be pairwise disjoint, we obtain $\mu=\# A \geq(l-1)(d+1)+d=l(d+1)-1$.

Now consider the case $d=1$. We have already shown that $\# A=\mu \leq(d+1) l-1=$ $2 l-1$. However, we have assumed that $\# V=n \geq 2 l$; thus $A \varsubsetneqq V$. Take a vertex $x \in V \backslash A ; x$ lies between two consecutive points of $A$, say between $a_{i}$ and $a_{i+1}$, for some $i, 1 \leq i \leq \mu$. Define $\bar{e}=\left[x, a_{i+d}\right]=\left[x, a_{i+1}\right]$ (directed from $x$ to $a_{i+1}$ ). The rest of the proof is exactly the same as in the previous case $d>1$. This concludes Case 1 $(A=B)$.

\section{Continuation}

Before passing to the case $A \neq B$, we prove two more propositions. Call an edge $\left[a_{i}, b_{i}\right] \in E^{*}$ long if $\beta_{i}>\alpha_{i}+1$ (i.e., if $\lambda\left(\left[a_{i}, b_{i}\right]\right)>1$ ). (Recall that $a_{i}=v\left(\alpha_{i}\right)$, $b_{i}=v\left(\beta_{i}\right), 0 \leq \alpha_{i}<n, \alpha_{i}<\beta_{i}<\alpha_{i}+n$.)

Proposition 5.1. If, for some $1 \leq i \leq \mu,\left[a_{i}, b_{i}\right] \in E^{*}$ is a long edge, then $a_{i} \in B$, $b_{i} \in A$.

Proof (see Fig. 2). Define $x=v\left(\beta_{i}-1\right), \bar{e}=\left[a_{i}, x\right] . \bar{e}$ lies on the right side of $e_{i}$, hence $\bar{e} \notin E$. Now put $\bar{E}=E \cup\{\bar{e}\}, \bar{G}=(V, \bar{E}), \bar{G}^{*}=\operatorname{Ext}(\bar{G})=\left(V, \bar{E}^{*}\right)$. As before, $\bar{E}^{*} \subset E^{*} \cup\{\bar{e}\}$. Since $G$ is $l$-saturated, $\bar{G}$, and therefore also $\bar{G}^{*}$, must include an $l$-cm $L . L$ necessarily consists of $\bar{e}$ and $l-1$ edges of $E^{*} \backslash\left\{e_{i}\right\} .\left(e_{i}\right.$ shares a vertex with $\bar{e}$.) All edges of $L \cap E^{*}$ lie strictly on the left side of $\bar{e}=\left[a_{i}, x\right]$. If no edge of $L \cap E^{*}$ uses the vertex $b_{i}$, then they all lie strictly on the left side of $e_{i}=\left[a_{i}, b_{i}\right]$, and

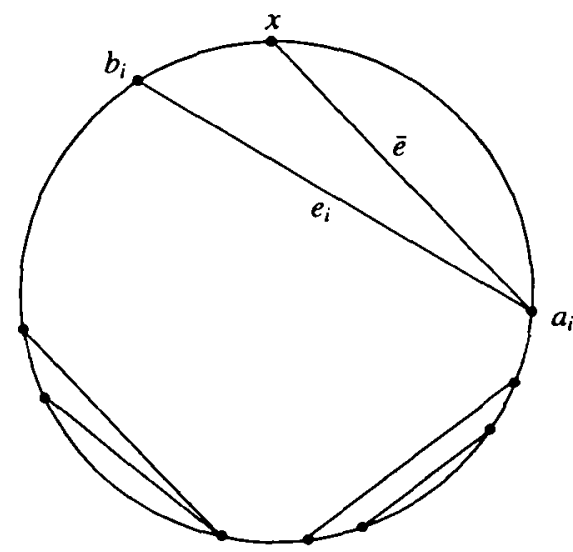

Fig. 2 


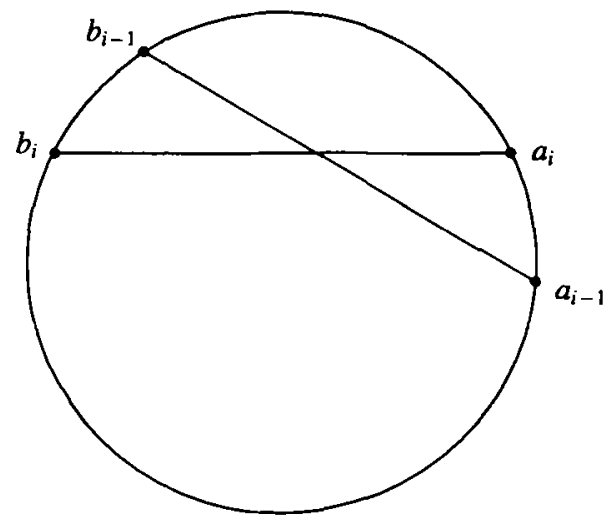

Fig. 3

then $L^{\prime}=(L \backslash\{\bar{e}\}) \cup\left\{e_{i}\right\}$ is an $l-\mathrm{cm}$ in $G^{*}$, which is impossible. Thus there is an edge $e^{\prime}=L \cap E^{*} \subset E^{*} \backslash\left\{e_{i}\right\}$ that uses $b_{i}$ as a vertex. Since two different edges of $E^{*}$ cannot have the same endpoint, $b_{i}$ must be the starting point of $e^{\prime}$, hence $b_{i} \in A$. The proof that $a_{i} \in B$ is entirely analogous and is left to the reader.

Proposition 5.2. If, for some $i, a_{i}$ lies strictly on the right side of $e_{i-1}$, then $a_{i+1}$ lies strictly on the right side of $e_{i}$.

Proof (see Fig. 3). If $a_{i}$ lies strictly on the right side of $e_{i-1}$, then $e_{i-1}$ must be a long edge, and therefore, by Proposition 5.1, $b_{i-1} \in A$. Since $e_{i}$ cannot be entirely on the right side of $e_{i-1}, b_{i}$ must lie strictly on the left side of $e_{i-1}$. Moving on bd $[V]$ counterclockwise from $a_{i}$, we reach $a_{i+1}$ either at $b_{i-1}(\in A)$ or even earlier; at any rate, before $b_{i}$. Thus $a_{i+1}$ lies strictly on the right side of $e_{i}$.

From Proposition 5.2 we conclude (by cyclical induction), that if, for some $i, a_{i}$ lies strictly on the right side of $e_{i-1}$, then the same holds for all $i$, and therefore all edges of $E^{*}$ are long, hence $A=B$ (by Proposition 5.1).

Case II: $A \neq B$. We assume henceforth that $a_{i}$ lies on the left side of $e_{i-1}$, for all $1 \leq i \leq \mu$. This implies (see (4.3))

$$
0 \leq \alpha_{1}<\beta_{1} \leq \alpha_{2}<\beta_{2} \leq \alpha_{3}<\beta_{3} \leq \cdots \leq \alpha_{\mu}<\beta_{\mu} \leq \alpha_{1}+n .
$$

(The rightmost inequality follows from the fact that $a_{1}\left(=a_{\mu+1}\right)$ lies on the left side of $e_{\mu}$.)

Thus $E^{*}$ is a convex set of edges of $\mathrm{CK}(n)$. If we put

$$
W=A \cup B=\bigcup\left\{\text { verte: } e \in E^{*}\right\},
$$

then $E^{*}$ is a set of boundary edges of [W]. [W] is a nondegenerate polygon, since \# $E^{*}=$ $\mu \geq 2$ (see the beginning of Section 4 ). $E^{*}$ does not contain all boundary edges of $[W]$, 
since $A \neq B$. Thus $E^{*}$ is the union of, say, $\kappa$ pairwise disjoint boundary paths $P_{1}, \ldots, P_{\kappa}$ of $[W]$. Assume these paths $P_{1}, \ldots, P_{k}$ appear in this order, counterclockwise, on the boundary of $[W]$, and assume also that each path $P_{i}$ is directed counterclockwise. Denote by $\mu_{i}$ the length (=number of edges) of $P_{i}$, and define $\lambda_{i}=\left[\frac{1}{2}\left(\mu_{i}+1\right)\right](i=1, \ldots, \kappa)$, $\lambda=\lambda_{1}+\cdots+\lambda_{k}$. Clearly, $\mu=\mu_{1}+\cdots+\mu_{\kappa}$, and the maximum size of a matching included in $P_{i}$ is $\lambda_{i}(i=1, \ldots, \kappa)$. It follows that the maximum size of a convex matching in $G$ is $\lambda$ (see Proposition 1.2). However, $G$ is $l$-saturated, and therefore the maximal size of a convex matching in $G$ must be $l-1$, i.e., $\lambda=l-1$. ( $G$ is not complete, since $n \geq 2 l$, and adding any edge to $G$ will produce an $l-\mathrm{cm}$.)

Proposition 5.3. $\mu_{i}=2 \lambda_{i}$ for $i=1, \ldots, \kappa$, and therefore $\mu=2 \lambda=2(l-1)$.

Proof. If, for some $i, \mu_{i} \neq 2 \lambda_{i}$, then $\mu_{i}=2 \lambda_{i}-1$. Let $\bar{e}$ be the edge that connects the front vertex of $P_{i}$ with the back vertex of $P_{i+1}$. $\left(P_{i+1}=P_{1}\right.$ if $i=\kappa$. If $\kappa=1$, then $P_{i}=P_{i+1}=P_{1}$.) $\bar{e}$ is a boundary edge of $[W]$, and $\bar{e} \notin E$. Define $\bar{G}=G \cup\{\bar{e}\}$. The set of extreme edges of $\bar{G}$ is $E^{*} \cup\{\bar{e}\} . \bar{G}$ is not $l$-free, and thus there is an $l$-cm with edges in $E^{*} \cup\{\bar{e}\}$. However, this is impossible.

If $\kappa>1$, then $E^{*} \cup\{\bar{e}\}$ consists of the paths $P_{1}, \ldots, P_{i} \cup\{\bar{e}\} \cup P_{i+1}, P_{i+2}, \ldots, P_{\kappa}$, and

$$
\begin{aligned}
{\left[\frac{1}{2}\left(1+\#\left(P_{i} \cup\{\bar{e}\} \cup P_{i+1}\right)\right)\right] } & =\left[\frac{1}{2}\left(1+\mu_{i}+1+\mu_{i+1}\right)\right] \\
& =\left[\frac{1}{2}\left(2 \lambda_{i}+1+\mu_{i+1}\right)\right] \\
& =\lambda_{i}+\left[\frac{1}{2}\left(1+\mu_{i+1}\right)\right]=\lambda_{i}+\lambda_{i+1} .
\end{aligned}
$$

Thus the addition of $\bar{e}$ does not increase the maximal size of a matching in $P_{i} \cup P_{i+1}$. If $\kappa=1$, then $E^{*}=P_{1}$, and $E^{*} \cup\{\bar{e}\}$ is a convex circuit of $2 \lambda$ edges (the whole boundary of $[W]$ ), which again has no matching of size larger than $\lambda=l-1$.

Proposition 5.4. $\quad E^{*}$ consists of boundary edges of $[V]$ only.

Proof. It suffices to show that $E^{*}$ contains no long edges.

Assume $e=[a, b]$ is a long edge in $E^{*}$, and let $x$ be a vertex of $V$, strictly on the right side of $e$. $e$ belongs to a path $P_{i}(1 \leq i \leq \kappa)$. Since $\# P_{i}=2 \lambda_{i}$ is even, $e$ divides $P_{i}$ into an even path and an odd path. Assume, e.g., that $P_{i}$ has $2 \alpha$ edges behind $a$ and $2 \beta-1$ edges in front of $b\left(1 \leq \beta \leq \lambda_{i} ; \alpha+\beta=\lambda_{i}\right)$. Define $\bar{e}=[x, b]$ and $\bar{G}=G \cup\{\bar{e}\}$. As before, $\bar{e} \notin G$, and therefore $\bar{G}$ must have an $l$-cm $L$ with edges in $E^{*} \cup\{\bar{e}\} . L$ must use $\bar{e}$, and therefore cannot use $e(e \cap \bar{e}=\{b\} \neq \emptyset)$. However, $\left(E^{*} \backslash\{e\}\right) \cup\{\bar{e}\}$ is obtained from $E^{*}$ by replacing the path $P_{i}$ of length $2(\alpha+\beta)$ by two disjoint paths $P_{i}^{\prime}, P_{i}^{\prime \prime}$ of lengths $2 \alpha$ and $2 \beta$, respectively. This replacement does not increase the size $\alpha+\beta$ of a maximal matching in $P_{i}$.

By now we know that $E^{*}$ consists of $\kappa$ (one or more) disjoint even paths on the boundary of $[V]$, of total length $2(l-1)$. In the next proposition we show that $\kappa>1$, 
and thus $E^{*}$ is indeed one of the sets $E^{*}$ (of type I) constructed in Section 2. (The evenness of $\# P_{i}=\mu_{i}=2 \lambda_{i}$ ensures (2.2), and $\kappa>1$ ensures (2.3).)

Proposition 5.5. $\kappa>1$.

Proof. Assume $\kappa=1$, i.e., $E^{*}=P_{1}$ is one long path of length $2(l-1)$ on the boundary of $[V]$, going from $a$ to $b$. Define $\bar{e}=[b, a]$ and $\bar{G}=\operatorname{Bet}\left(V, E^{*} \cup\{\bar{e}\}\right)$. Then $\bar{G} \supseteq G$, since $\bar{e} \notin E$. However, $\bar{G}$ is one of the $l$-saturated graphs of type II constructed in Section 3 (with $d=1, m=(d+1) l-1=2 l-1$, and $A$ the set of vertices of the path $\left.P_{1}\right)$. Thus $\bar{G}$ is $l$-free, and therefore $G$ is not $l$-saturated.

Conclusion 5.6. Let $G=(V, E)$ be an $l$-saturated cgg, where $\# V=n \geq 2 l \geq 4$. Then $G$ is either of type I or of type $I$.

Remark 5.7. The last conclusion together with Proposition 3.2(b) implies that any 2 -saturated $\operatorname{cgg}$ on $n$ vertices has precisely $n$ edges (and is of type II; see Remark 3.3(ii) above).

\section{Determination of $\mathrm{cm}_{l}(n)$}

We defined $\mathrm{cm}_{l}(n)$ in the Introduction to be the minimum number of edges of an $l$ saturated cgg with $n$ vertices. Recalling Remark $2.1, \mathrm{~cm}_{1}(n)=\mathrm{CM}_{1}(n)=0((V, \emptyset)$ is 1-saturated). All 2-saturated cgg's with $n \geq 4$ vertices are of type II (see Conclusion 5.6 and the Remark after (2.3)), and they all have exactly $n$ edges (see Proposition 3.2(b) and Remarks 3.3(ii) and 5.7). Thus $\mathrm{cm}_{2}(n)=\mathrm{CM}_{2}(n)=n$ for $n \geq 4$ (actually for $n \geq 3$ ).

As for small $n, \mathrm{~cm}_{l}(n)=\mathrm{CM}_{l}(n)=\left(\begin{array}{l}n \\ 2\end{array}\right)$ if $n<2 l(\mathrm{CK}(n)$ is $l$-saturated), and, for $n=2 l(\geq 4), \mathrm{cm}_{l}(n)=\mathrm{CM}_{l}(n)=\left(\begin{array}{l}n \\ 2\end{array}\right)-2$. (All $l$-saturated cgg's with $n=2 l$ vertices have $\left(\begin{array}{l}n \\ 2\end{array}\right)-2$ edges, and the two missing edges lie on the boundary; they are of type I if the missing edges are adjacent, and of type II if not. In the latter case the missing edges separate bd $[V]$ into two even paths.)

Assume therefore henceforth that $n>2 l \geq 6$.

Denote by $\mathrm{P}_{l . n}(d)$ the minimum number of edges of an $l$-saturated cgg of type II with $n$ vertices and jump number $d$. By Proposition 3.2(a)

$$
\mathrm{P}_{l, n}(d)=\left(\begin{array}{l}
n \\
2
\end{array}\right)-m(d-1)-2 d(n-m)+\frac{1}{2}(n-m)-\frac{1}{2}(n-m)^{2},
$$

where $d \geq 1$ and $m=(d+1) l-1 \leq n$, hence $1 \leq d \leq[(n+1) / l]-1$. Substituting, we obtain

$$
\begin{aligned}
\mathbf{P}_{l, n}(d)=\left(\begin{array}{l}
n \\
2
\end{array}\right)-( & (d+1) l-1)(d-1)-\left(2 d-\frac{1}{2}\right) \\
& \times(n+1-(d+1) l)-\frac{1}{2}(n+1-(d+1) l)^{2} .
\end{aligned}
$$


This is a quadratic polynomial in $d$, say $a d^{2}+b d+c$, where $a=l-\frac{1}{2} l^{2}=\frac{1}{2} l(2-l)<0$ (since $l \geq 3$ ) and

$$
b=n l-l^{2}-2 n+2 \frac{1}{2} l-1 .
$$

This is a strictly concave function of $d$. It attains its maximum at the point $d=-b / 2 a$, and is symmetric with respect to this point. It attains its minimum on the effective range of $d, 1 \leq d \leq[(n+1) / l]-1$, at one of the two endpoints. Note that if $n<3 l-1$, then $[(n+1) / l]=2$, and the effective range of $d$ is just $d=1$. To show that $P_{l, n}(d)$ attains its minimum at the left endpoint $d=1$ (and only there), it suffices to show that the point of symmetry of $\mathrm{P}_{l, n}(d),-b / 2 a$, lies strictly to the right of the center of the effective interval, i.e.,

$$
-\frac{b}{2 a}>\frac{1}{2}\left(1+\left[\frac{n+1}{l}\right]-1\right)=\frac{1}{2}\left[\frac{n+1}{l}\right] .
$$

Since $a<0$, it is enough to show that

$$
b>-a\left(\frac{n+1}{l}\right) \quad\left(=\frac{1}{2}(l-2)(n+1)\right) .
$$

Using (6.2) and some simple manipulations, we find that (6.3) is equivalent to $(n-2 l)(l-2)>0$, which is true.

Thus we have shown that

$$
\begin{aligned}
\min \left\{\mathrm{P}_{l, n}(d): 1 \leq d \leq\left[\frac{n+1}{l}\right]-1\right\} & =\mathrm{P}_{l, n}(1) \quad(\text { see }(6.1)) \\
& =\left(\begin{array}{l}
n \\
2
\end{array}\right)-\frac{3}{2}(n+1-2 l)-\frac{1}{2}(n+1-2 l)^{2} \\
& =(2 l-3)(n-l+1)+1 .
\end{aligned}
$$

The minimum is attained at $G$ (of type II) iff $G$ has jump number $d=1$ and, say, $x_{1}+x_{2}=n+1-2 l, x_{3}=\cdots=x_{2 l-1}=0$ (see text after (3.1)). This characterizes the minimal $l$-saturated cgg's.

By Proposition 2.4, the minimum number of edges for cgg's of type $I$ is $2(l-1) n-$ $\left(2 l^{2}-3 l+2\right)$, which is larger (by $\left.n-2 l\right)$ than (6.4).

Combining all the previous considerations, we obtain:

\section{Theorem 6.1.}

$$
\mathrm{cm}_{l}(n)=\left\{\begin{array}{lll}
\left(\begin{array}{l}
n \\
2
\end{array}\right) & \text { if } n<2 l \\
0 & \text { if } l=1 \\
n & \text { if } n \geq 2 l=4 \quad \text { (i.e., } l=2) \\
(2 l-3)(n-l+1)+1 & \text { if } n \geq 2 l \geq 6 \quad \text { (i.e., } l \geq 3) .
\end{array}\right.
$$

\section{Determination of $\mathrm{CM}_{l}(n)$}

We defined $\mathrm{CM}_{l}(n)$ in the Introduction to be the maximum number of edges of an $l$ saturated cgg with $n$ vertices, or, equivalently, the maximum number of edges of an 
$l$-free $\operatorname{cgg}$ with $n$ vertices. At the beginning of Section 6 we have determined $\mathrm{CM}_{l}(n)$, and described the extremal cgg's, in the following cases: $\mathrm{CM}_{1}(n)=\mathrm{cm}_{1}(n)=0$, $\mathrm{CM}_{2}(n)=\mathrm{cm}_{2}(n)=n$ for $n \geq 4, \mathrm{CM}_{l}(n)=\mathrm{cm}_{l}(n)=\left(\begin{array}{l}n \\ 2\end{array}\right)$ if $n<2 l$, and $\mathrm{CM}_{l}(2 l)=$ $\mathrm{cm}_{l}(2 l)=\left(\begin{array}{l}2 l \\ 2\end{array}\right)-2$ for $l \geq 2$. Assume, henceforth, that $n \geq 2 l \geq 6$.

In Proposition 2.3 we have a formula (2.4) for the maximum number of edges in an $l$-saturated cgg of type I with $n$ vertices $(n \geq 2 l \geq 6)$. Call this number $\varphi_{l}(n)$ $\left(=\left(\begin{array}{l}n \\ 2\end{array}\right)-(l-1)\left(\begin{array}{l}q \\ 2\end{array}\right)-r q\right.$, where $\left.n=(l-1)(q+1)+r, 1 \leq r \leq l-1\right)$.

As we noted in Section 2, (2.4) also holds for $r=0$. It follows by an easy computation that, for $n$ as above (with $r \geq 1$ ),

$$
\varphi_{l}(n)=\varphi_{l}(n-1)+n-1-q,
$$

provided, of course, that $n>2 l$. As we mentioned in Section $2, \varphi_{l}(n)=\mathrm{T}_{l}(n)+n-l+1$.

Our purpose in this section is to show that

$$
\mathrm{CM}_{l}(n)=\varphi_{l}(n)
$$

for $n \geq 2 l \geq 6$. This is done by induction on $n$, for any fixed $l \geq 3$, starting with $n=2 l$, in the same spirit as the usual proof of Turán's theorem. To make the induction step work we must know that every $l$-free $\mathrm{cgg}$ has a vertex of sufficiently low valence. This is provided by the following lemma.

Lemma 7.1. Let $G=(V, E)$ be a cgg of order $n$. Assume that $6 \leq 2 l \leq n=$ $(l-1)(q+1)+r, 1 \leq r \leq l-1$. If every vertex of $G$ has valence $\geq n-q$, then $G$ includes an $l-c m$.

Proof. Orient the boundary of $[V]$ counterclockwise. For each vertex $v$, denote by $v^{+}$ the first vertex after $v$ that is adjacent to $v$ in $G$ and by $v^{-}$the last vertex before $v$ that is adjacent to $v$.

Define $\alpha(v)=1+\lambda\left(\left[v, v^{+}\right]\right), \beta(v)=1+\lambda\left(\left[v^{-}, v\right]\right)$, i.e., $\alpha(v)$ (resp. $\left.\beta(v)\right)$ is the number of vertices of $G$ that lie in the closed half-plane to the right of $\left[v, v^{+}\right]$(resp. $\left.\left\lfloor v^{-}, v\right]\right)$.

Clearly, $\alpha(v), \beta(v) \geq 2$, and $v$ is not adjacent to any vertex that lies strictly between $v^{-}$and $v^{+}$, including $v$ itself. The number of these vertices is $\alpha(v)+\beta(v)-3$. Therefore,

$$
\operatorname{val}(v, G) \leq n-\alpha(v)-\beta(v)+3
$$

Since, by assumption, $\operatorname{val}(v, G) \geq n-q$, we conclude that

$$
\alpha(v)+\beta(v) \leq q+3,
$$

and therefore $\alpha(v), \beta(v) \leq q+1$ for all $v \in V$.

Consider three cases:

(I) $\alpha(v) \leq \beta(v)$ for all $v \in V$.

(II) $\alpha(v)>\beta(v)$ for all $v \in V$.

(III) Neither I nor II. 
Case I. For each vertex $v \in V$, denote by $v^{\prime}$ the immediate counterclockwise successor of $v$ along the boundary of $[V]$. Choose an arbitrary vertex of $G$, call it $v_{1}$, and define vertices $v_{2}, v_{3}, \ldots, v_{l}$ inductively by

$$
v_{i+1}=\left(v_{i}^{+}\right)^{\prime} \quad(i=1, \ldots, l-1) .
$$

The closed boundary arc of $[V]$ to the right of $\left[v_{i}, v_{i}^{+}\right]$contains $\alpha\left(v_{i}\right)$ vertices of $G$. These boundary arcs come in a continuous succession one after another. If we show that $\sum_{i=1}^{l} \alpha\left(v_{i}\right) \leq n$, it follows that the edges $\left[v_{i}, v_{i}^{+}\right], i=1, \ldots, l$, form an $l-\mathrm{cm}$. Since we are in case $\mathrm{I}$, and in view of $(7.3)$, we find that $\alpha\left(v_{i}\right) \leq[(q+3) / 2]$ for all $i$, hence

$$
\sum_{i=1}^{l} \alpha\left(v_{i}\right) \leq l\left[\frac{q+3}{2}\right]
$$

If $q \leq 2$, then $[(q+3) / 2] \leq 2$ and therefore $l[(q+3) / 2] \leq 2 l \leq n$. If $q \geq 3$, then

$$
l\left[\frac{q+3}{2}\right] \leq l \frac{q+3}{2} \stackrel{(*)}{\leq}(q+1)(l-1)+1 \leq(q+1)(l-1)+r=n .
$$

(The inequality (*) holds easily from the assumptions $q \geq 3, l \geq 3$, please check.)

Case II. This case is impossible. If $e=[v, w]$ is a directed edge of $G$ that minimizes $\lambda(e)$ (over all edges of $G$ ), then clearly $\beta(v) \geq \alpha(v)$.

Case III. In this case there must be a vertex $v$ with $\alpha(v)>\beta(v)$, whose successor $v^{\prime}$ satisfies the opposite inequality $\alpha\left(v^{\prime}\right) \leq \beta\left(v^{\prime}\right)$.

Define $v_{1}=v, v_{2}=v^{\prime}$, and continue by induction as in Case I:

$$
v_{i+1}=\left(v_{i}^{+}\right)^{\prime} \quad \text { for } \quad i=2,3, \ldots, l-1 .
$$

Consider the edges $\left[v_{1}^{-}, v_{1}\right],\left[v_{2}, v_{2}^{+}\right],\left[v_{3}, v_{3}^{+}\right], \ldots,\left[v_{l}, v_{l}^{+}\right]$. The closed boundary arcs to the right of these edges form a continuous succession, exactly as in Case $I$. The number of vertices of $G$ on these arcs is $\beta(v)$ for the first edge, $\alpha\left(v^{\prime}\right)$ for the second one, and $\alpha\left(v_{i}\right)$ for $3 \leq i \leq l$.

As in Case I, it suffices to show that $\beta(v)+\alpha\left(v^{\prime}\right)+\sum_{i=3}^{l} \alpha\left(v_{i}\right) \leq n$. From our assumptions $\left(\alpha(v)>\beta(v), \alpha\left(v^{\prime}\right) \leq \beta\left(v^{\prime}\right)\right)$ it follows that $\beta(v)<(q+3) / 2, \alpha\left(v^{\prime}\right) \leq$ $(q+3) / 2$, and thus $\beta(v)+\alpha\left(v^{\prime}\right)<q+3$, i.e., $\leq q+2$.

For $3 \leq j \leq l$ we have $\alpha\left(v_{j}\right) \leq q+1$. Summing together we find that $\beta(v)+\alpha\left(v^{\prime}\right)+$ $\sum_{i=3}^{l} \alpha\left(v_{i}\right) \leq q+2+(l-2)(q+1)=(l-1)(q+1)+1 \leq n$.

Theorem 7.2. For $n \geq 2 l \geq 6, \mathrm{CM}_{l}(n)=\mathrm{T}_{l}(n)+n-l+1$. (The extremal graphs are determined later in Section 8.)

Proof. As we mentioned earlier in this section, $\mathrm{T}_{l}(n)+n-l+1$ is equal to $\varphi_{l}(n)$, which is the right-hand side of (2.4). By Proposition 2.3, there is an $l$-saturated cgg (of type I) with $n$ vertices and $\varphi_{l}(n)$ edges. Thus $\mathrm{CM}_{l}(n) \geq \varphi_{l}(n)$.

To prove the opposite inequality we proceed by induction on $n(n \geq 2 l)$ for any fixed 
$l \geq 3$. When $n=2 l(=(l-1)(1+1)+2)$ we already know that $\mathrm{CM}_{l}(n)=\left(\begin{array}{l}n \\ 2\end{array}\right)-2$, and this agrees with the value of $\varphi_{l}(n)$, as defined in the beginning of this section.

The induction step $(n-1 \rightarrow n)$ : Suppose $G$ is an $l$-free cgg of order $n, n>2 l \geq 6$. Assume $n=(l-1)(q+1)+r, 1 \leq r \leq l-1$. By Lemma 7.1, $G$ has a vertex $v$ of valence $\leq n-q-1$. Define $G^{\prime}=G \backslash v$. $G^{\prime}$ is an $l$-free $\operatorname{cgg}$ of order $n-1$, $n-1 \geq 2 l \geq 6$, and therefore, by the induction hypothesis $e\left(G^{\prime}\right) \leq \varphi_{l}(n-1)$. Hence, by $(7.1), e(G)=e\left(G^{\prime}\right)+\operatorname{val}(v, G) \leq \varphi_{l}(n-1)+n-q-1=\varphi_{l}(n)$.

\section{Determination of the $l$-Extremal cgg's}

We start with the cases that are already settled. Note that whenever $\mathrm{CM}_{l}(n)=\mathrm{cm}_{l}(n)$ the notions of " $l$-saturated" and " $l$-extremal" become the same.

Case I: $l=1, n \geq 2 . \mathrm{CM}_{1}(n)=\mathrm{cm}_{1}(n)=0$, and the extremal cgg consists of $n$ vertices in convex position and no edges.

Case $\mathrm{II}: l=2, n \geq 4 . \mathrm{CM}_{2}(n)=\mathrm{cm}_{2}(n)=n$.

The extremal cgg's have been determined in Remark 3.3(ii). They are all of type II and can be described as follows: Start with a convex $n$-gon $P$ (vert $P=V$ ). For some $d, 1 \leq d \leq[(n-1) / 2]$, choose (arbitrarily) some $2 d+1$ vertices of $P$ (the set $A$ ), and denote their convex hull by $Q$. Draw all $2 d+1$ main diagonals (=diagonals of order $d$ ) of $Q$. Finally connect each vertex $q$ of $Q$ with the vertices of $P$ that lie within the angle formed by the two main diagonals of $Q$ emanating from $q$.

Case III: $1 \leq n<2 l$. Here $\mathrm{CM}_{l}(n)=\mathrm{cm}_{l}(n)=\left(\begin{array}{l}n \\ 2\end{array}\right)$, and the extremal cgg is CK$(n)$.

Case IV: $n=2 l \geq 4$. Here $\mathrm{CM}_{l}(2 l)=\mathrm{cm}_{l}(2 l)=\left(\begin{array}{c}2 l \\ 2\end{array}\right)-2$, and the extremal cgg's are obtained from $\mathrm{CK}(2 l)$ by deleting two boundary edges $e$ and $e^{\prime}$ that are at an odd distance apart.

If $e$ and $e^{\prime}$ are not adjacent, then they separate the boundary of [V] into two nondegenerate even paths, and the cgg obtained is of type I (with $x_{i}=2$ twice, and $x_{i}=1$ $l-3$ times). This can happen only when $l \geq 3$.

If $e$ and $e^{\prime}$ are adjacent, then we obtain a cgg of type II, with $d=1, x_{i}=1$ once and $x_{i}=02 l-2$ times. We call this graph the $(l, 2 l)$-exceptional cgg.

Case V: Suppose $n \geq 2 l \geq 6$, and let $G$ be an $l$-saturated cgg of order $n$ of type I, as described in Section 2, with sets $X_{i}$ of size $x_{i}, i=1, \ldots, l-1, x_{i} \geq 1$ for all $i, x_{i} \geq 2$ at least twice, and $\sum x_{i}=n-l+1$. Assume, also, as usual, that $n=(l-1)(q+1)+r$, $q \geq 1,1 \leq r \leq l-1$, and if $q=1$, then $r>1$.

We have seen (Proposition 2.3 and Theorem 7.2) that $G$ is extremal iff the numbers $x_{i}$ are nearly equal, i.e., $x_{i}=q+1 r$ times and $x_{i}=q l-1-r$ times.

The graphs satisfying this condition are call equipartitioned.

Case VI: Let $l=3, n=7$. Consider the 3-saturated cgg of order 7, of type II with $d=1$ (hence $m=5)$ and $\left(x_{1}, x_{2}, x_{3}, x_{4}, x_{5}\right)=(1,0,1,0,0)$. This one can also be described (simpler) as a $\mathrm{CK}(7)$ with two disjoint boundary paths of length 2 removed. It has 17 $\left(=\left(\begin{array}{l}7 \\ 2\end{array}\right)-4\right)$ edges, and is therefore extremal, since $\varphi_{3}(7)=17$. We call this graph the $(3,7)$-exceptional cgg. 
Theorem 8.1. Suppose $n \geq 2 l \geq 6$. Then the only l-extremal cgg's of order $n$ are those listed above, i.e.:

(a) The equipartitioned l-saturated cgg's of type I of order $n$.

(b) For $n=2 l$, the $(l, 2 l$ )-exceptional cgg's (of type II) mentioned in Case IV.

(c) For $l=3, n=7$, the (3,7)-exceptional cgg of type II described in Case VI.

Proof. Every $l$-extremal cgg is, a fortiori, $l$-saturated. By Conclusion 5.6, every $l$ saturated cgg of order $n(n \geq 2 l \geq 6)$ is either of type I or of type II. If it is of type I, then we already know (Proposition 2.3 and Theorem 7.2) that it is $l$-extremal iff it is equipartitioned. Thus it only remains to show that if $G$ is an $l$-saturated $\operatorname{cgg}$ of order $n$ of type II, and $G$ is not one of the exceptional cgg's, then $e(G)<\mathrm{CM}_{l}(n)$. This is precisely what we are going to do.

For small $n$, i.e., for $n \leq 8$ if $l=3$, and for $n \leq 2 l+1$ if $l \geq 4$, we use a direct approach. After having cleared the excepotional cgg's out of the picture, we continue by induction on $n$, for each fixed $l$.

The case $n=2 l \geq 6$ is already settled. We know all $l$-saturated ( $=l$-extremal) cgg's of order $2 l$ (Case IV above). One of them is the $(l, 2 l$ )-exceptional cgg (of type II), and the remaining ones are of type I, equipartitioned. (There are precisely [ $(l-1) / 2]$ nonisomorphic cgg's of this kind.)

Next come the cases $l=3, n=7,8$. If $n=7$, then from $n \geq m=(d+1) l-1$ it follows that $d$ must be $1, m=5$, and $\sum_{i=1}^{5} x_{i}=n-m=2$. When $\left(x_{1}, x_{2}, x_{3}, x_{4}, x_{5}\right)=$ $(1,0,1,0,0)$ we obtain the $(3,7)$-exceptional cgg, with 17 edges. In the remaining cases, where $\left(x_{1}, x_{2}, x_{3}, x_{4}, x_{5}\right)$ is either $(1,1,0,0,0)$ or $(2,0,0,0,0)$, the two $X$-vertices are not joined by an edge, and we are left with only 16 edges.

For $n=8$, we have $\mathrm{CM}_{3}(8)=\left(\begin{array}{l}8 \\ 2\end{array}\right)-6=22$.

The possibilities for 3-saturated cgg's of type II with eight vertices are: $d=2, m=8$, $n-m=0$. This is CK(8) with all boundary edges removed, and the number of edges is $\left(\begin{array}{l}8 \\ 2\end{array}\right)-8=20<22$. Or $d=1, m=5, \sum_{i=1}^{5} x_{i}=n-m=3$. Each $X$-vertex is not joined to the two $A$-vertices that flank its block $X_{i}$.

Moreover, two $X$-vertices that belong to the same block $X_{i}$, or to adjacent blocks, are not joined to each other. Since a pentagon does not have three mutually nonadjacent edges, we lose at least seven $(=3 \times 2+1)$ edges, i.e., $e(G) \leq\left(\begin{array}{c}8 \\ 2\end{array}\right)-7=21$.

Finally consider the case $n=2 l+1, l \geq 4$. Here a type $\Pi$ cgg must have $d=1$, since $d=2$ would lead to $m=3 l-1$, which is $>2 l+1$. Thus $m=2 l-1$, and $\sum_{i=1}^{n} x_{i}=n-m=2$. As mentioned before, each $X$-vertex is not joined to exactly two $A$-vertices, and therefore $e(G) \leq\left(\begin{array}{c}2 l+1 \\ 2\end{array}\right)-4$. However, $\mathrm{CM}_{l}(2 l+1)=\left(\begin{array}{c}2 l+1 \\ 2\end{array}\right)-3$ for $l \geq 4$.

Now comes the inductive step $n-1 \rightarrow n$. We assume that either $l=3$ and $n \geq 9$, or $l \geq 4$ and $n \geq 2 l+2$. By the induction hypothesis, all $l$-extreme cgg's of order $n-1$ are of type $I$ and equipartitioned.

Let $\tilde{G}=(\tilde{V}, \tilde{E})$ be an $l$-saturated $\operatorname{cgg}$ of order $n$ and of type II. We assume that $\tilde{G}$ is $l$-extreme, i.e., $e(\tilde{G})=\mathrm{CM}_{l}(n)$, and this leads us to a contradiction.

Write $n=(l-1)(q+1)+r$, where $1 \leq r \leq l-1$. By Lemma 7.1, $\tilde{G}$ has a vertex of valence $\leq n-q-1$. Pick a vertex $v$ of minimal valence in $\tilde{G}$ and define $V=\tilde{V} \backslash\{v\}$, $G=\tilde{G} \backslash v . G$ is $l$-free, of order $n-1$, and therefore $e(G) \leq \mathrm{CM}_{l}(n-1)$. 
Thus $e(\tilde{G})=\operatorname{val}(v, \tilde{G})+e(G) \leq n-q-1+\mathrm{CM}_{l}(n-1)=\mathrm{CM}_{l}(n)=e(\tilde{G})($ see (7.1) and (7.2)).

It follows that $\operatorname{val}(v, \tilde{G})=n-q-1$, and $e(G)=\mathrm{CM}_{l}(n-1)$, i.e., $G$ is also $l$-extreme. By the induction hypothesis $G$ is of type $I$ and equipartitioned.

In particular, $G$ has exactly $2(l-1)$ extreme edges, and these form a number (at least two) of disjoint paths of even length on the boundary of $[V]$. Thus all extreme edges of $G$ are "short" (using the language of Section 5).

On the other hand, if $e$ is an extreme edge of $\tilde{G}$ that does not involve the vertex $v$, then $e$ is obviously also an extreme edge in $G(=\tilde{G} \backslash v)$. Thus all but at most two of the extreme edges of $\tilde{G}$ are extreme also in $G$.

If $d>1, \tilde{G}$ has $m$ extreme edges, where $m=(d+1) l-1 \geq 8$, and none of them is a boundary edge of $[\tilde{V}]$. Removing $v$, we lose at most two of these edges, and at most another one becomes a boundary edge of [V] (this can happen only if $d=2$ ). Thus $G$ has at least five "long" extreme edges, which is impossible.

The case $d=1$ remains. Here the extreme edges of $\tilde{G}$ form a convex circuit of length $2 l-1$. If the removed vertex $v$ is an $X$-vertex, then no extreme edge is lost and thus $G$ has a circuit of extreme edges, which is impossible. Assume therefore that the removed vertex $v$ is an $A$-vertex, say $v=a_{1}$. (Here we start using the notation of Section 3, and the contents of Proposition 3.1.)

If $X_{1} \cup X_{m} \neq \emptyset$, then $\operatorname{val}\left(a_{1}, \tilde{G}\right)>\operatorname{val}(x, \tilde{G})$ for all $x \in X_{1} \cup X_{m}$, contrary to our assumption that $v$ is a vertex of minimal valence in $\tilde{G}$.

If $X_{1} \cup X_{m}=\emptyset$, then $a_{1}$ is joined in $\tilde{G}$ to all other vertices, which contradicts our assumption of minimal valence even stronger.

\section{Extremal Theory for Convex Sets of Edges}

In the preceding sections we developed the extremal theory for convex $l$-matchings in cgg's of order $n$. In this section we briefly present the corresponding theory for convex sets of $l$ (not necessarily disjoint) edges. We denote such sets by $l$-c. " $l$-c-free," " $l$-c-saturated," and " $l$-c-extremal" cgg's are defined exactly as in the Introduction, with " $l-\mathrm{cm}$ " replaced by " $l-\mathrm{c} . "$ The functions that correspond to $\mathrm{CM}_{l}(n)$ and $\mathrm{cm}_{l}(n)$ are denoted by $C_{l}(n)$ and $c_{l}(n)$.

As we already mentioned in Remark 1.5 , if there is an $l$-c in $G$, then there is already one in $\operatorname{Ext}(G)$. It follows that if $G$ is $l$-c-saturated, then $G=\operatorname{Bet}(G)=\operatorname{Bet}(\operatorname{Ext} G)$, as in Proposition 1.4(b).

The results can be summarized as follows:

\subsection{Results Concerning $c_{l}(n)$, Minimal l-c-Saturated cgg's, $\mathrm{C}_{l}(n)$, and l-c-Extremal cgg's}

(a) $n<l$, or $n=l \leq 2$. Every cgg of order $n$ is $l$-c-free, and thus $\mathrm{C}_{l}(n)=\mathrm{c}_{l}(n)=\left(\begin{array}{l}n \\ 2\end{array}\right)$. Extremal cgg: $\operatorname{CK}(n)$.

(b) $l=1 . \mathrm{C}_{1}(n)=\mathrm{c}_{1}(n)=0$. Extremal cgg: $\overline{\mathrm{CK}}(n)$ ( $n$ vertices, no edges).

(c) $l=2, n>2 . c_{2}(n)=1$. Unique minimal 2-c-saturated cgg: $n$ vertices, one boundary edge. 
$\mathrm{C}_{2}(n)=[n / 2]$. Unique 2-c-extremal cgg: $n$ vertices, [ $\left.n / 2\right]$ mutually intersecting, vertex disjoint edges. If $n$ is odd, there is one isolated vertex.

(d) $l \geq 3, n=l$. $\mathrm{c}_{l}(l)=\mathrm{C}_{l}(l)=\left(\begin{array}{l}l \\ 2\end{array}\right)-1$. Unique extremal cgg: $\mathrm{CK}(l)$ with one boundary edge removed. ((d) is included in (e).)

(e) $l \geq 3, n \geq l$.

$$
c_{l}(n)=\left(\begin{array}{l}
n \\
2
\end{array}\right)-\left(\begin{array}{c}
n-l+2 \\
2
\end{array}\right) .
$$

Unique minimal $l$-c-saturated cgg: Let $\mathrm{CK}(n)$ be a complete $\mathrm{cgg}$ based on a convex $n$-gon $P$. Let $B=\left\{b_{1}, b_{2}, \ldots, b_{n-l+2}\right\}$ be a set of $n-l+2$ consecutive vertices of $P$. Remove from $\operatorname{CK}(n)$ all $\left(\begin{array}{c}n-l+2 \\ 2\end{array}\right)$ edges that connect $B$-vertices.

$\mathrm{C}_{l}(n)=\mathrm{T}_{l}(n)$. Extremal cgg's: Turán graphs, where each of the $l-1$ maximal independent sets (of size $[n /(l-1)]$ or $[n /(l-1)\rceil$ appears as an arc of consecutive vertices on the boundary of the convex $n$-gon $P$. The extremal $\mathrm{cgg}$ is unique (up to isomorphism of cgg's) iff $n$ is congruent to 0,1 , or -1 modulo $l-1$.

\subsection{Characterization of $l$-c-Saturated $c g g$ 's}

(a) $l=2, n \geq 3$. Let $P$ be a convex $n$-gon with vertex set $V$. A $\operatorname{cgg} G=(V, E)$ is 2 -cfree iff the edges in $E$ are mutually intersecting and vertex-disjoint. Assume $\# E=m$. Then $m \leq[n / 2]$, and if $m>0$, then the vertices of the edges in $E$ divide the boundary of $P$ into $2 m$ pairwise disjoint open arcs. Denote these arcs by $V_{1}, V_{2}, \ldots, V_{2 m}$, in their natural cyclic order on bd $P$. Then $G$ is 2-c-saturated iff for each $i, 1 \leq i \leq n$, at least one of the two opposite arcs $V_{i}, V_{m+i}$ is an open edge of $P$ (i.e., contains no vertex of $P$ ).

(b) $l \geq 3, n \geq l$. Let $P$ be a convex $n$-gon with vertex set $V=\{v(0), \ldots, v(n-1)\}$, as in Section 1. Choose an integer $\delta$ (the jump number), $0 \leq \delta \leq(n+1) / l-1$. Define $m=(\delta+1) l-1(m \leq n)$. Choose a subset $A \subset V$ of size $m$. Assume that the points of $A$ appear in the counterclockwise cyclic order $a_{1}, \ldots, a_{m}, a_{1}$ on the boundary of $P$, and extend their indexing modulo $m$ to all integers. For $a \in A$, denote by $a^{\prime}$ the vertex of $P$ (not necessarily in $A$ ) that follows $a$ in the counterclockwise cyclic order. (If $a=v(v)$, then $a^{\prime}=v(v+1)$.)

Define $E^{*}=\left\{\left[a_{i},\left(a_{i+\delta}\right)^{\prime}\right]: i=1, \ldots, m\right\}, G^{*}=\left(V, E^{*}\right), G=\operatorname{Bet}\left(G^{*}\right)$. Then $G$ is $l$-c-saturated, and every $l$-c-saturated $\operatorname{cgg}$ on $V$ is obtained in this way.

\subsection{Proofs}

The verification of claims (a)-(d) in Section 9.1 and (a) in Section 9.2 is entirely straightforward and is left to the reader. Next come the claims regarding $C_{l}(n)$ and the extremal cgg's in (e) of Section 9.1. These can be tackled as follows: If a cgg $G$ on $n$ vertices has more than $\mathrm{T}_{l}(n)$ edges, then $G$ includes a $\mathrm{CK}(l)$, and the boundary edges of this $\mathrm{CK}(l)$ form an $l$-c. The same holds if $e(G)=\mathrm{T}_{l}(n)$, but $G$ is not isomorphic (as an abstract graph) to the appropriate Turán graph. (= complete $(l-1)$-partite graph on $n$ vertices with nearly equal color sets.) If $G$ is isomorphic to the Turán graph, but (at least) one of 
the color sets is not consecutive, then $G$ includes a convex circuit of length $l$ or $l+1$. (If color set $C$ is split, choose two appropriate vertices, $c, c^{\prime}$ for color $C$, separate them on both sides by two vertices $b, b^{\prime}$ not of color $C$, and add one representative of each other color class. This results in a set of $l$ or $l+1$ vertices, where each vertex is flanked by two vertices of a different color.)

If $G$ is a Turán graph, and all color sets are consecutive, then $G$ is $l$-c-free. (If $\left\{e_{1}, \ldots, e_{r}\right\}$ is a convex set of edges in $G, r \geq 2$, then beyond each edge $e_{i}$ there is a boundary edge whose two endpoints have different colors.)

We defer the proof of the claims in (e) of Section 9.1 that concern $c_{l}(n)$ and the minimal graphs until after the proof of (b) in Section 9.2.

Proof of (b) in Section 9.2. We must show that the cgg's $G$ constructed in (b) of Section 9.2 are $l$-c-free, that they are $l$-c-saturated, and that there are no other $l$-c-saturated cgg's on $V$. We model the proof of the first two of these three claims after the proof of Proposition 3.1, and only indicate the necessary changes.

Claim a. $\operatorname{Ext}(G)=G^{*}$.

If $\delta=0$, then $E^{*}$ is a set of $m=l-1$ boundary edges of $P$, which clearly implies $\operatorname{Ext}(G)=G^{*}$.

If $\delta>0$, we claim that the $m$ edges $\left[a_{i},\left(a_{i+\delta}\right)^{\prime}\right]$ are all distinct, and that none of them lies on the right side of any other one. Note that $\left(a_{i+\delta}\right)^{\prime}$ may or may not be equal to $a_{i+\delta+1}$. For $\left(a_{j},\left(a_{j+\delta}\right)^{\prime}\right](i<j<i+m)$ to be equal to $\left[a_{i},\left(a_{i+\delta}\right)^{\prime}\right]$, or lie on the right side of $\left[a_{i},\left(a_{i+\delta}\right)^{\prime}\right]$, we must have $i<j \leq i+\delta+1$ and $j+\delta+1 \geq i+m$, hence $2(\delta+1) \geq m$. However, $l \geq 3$ and $\delta>0$ imply $2(\delta+1)<l(\delta+1)-1=m$.

Claim b. An edge $e=[p, q]$ of $\mathrm{CK}(n)$ belongs to $G$ iff each "half-open side" of $e$ contains at least $\delta+1$ points of $A$.

Here an "half-open side" of $e=[p, q]$ means the boundary arc $[p \rightarrow q)$ of $P$ going counterclockwise from $p$ to $q$, including $p$, excluding $q$, or the complementary arc $[q \rightarrow p)$, going from $q$ (included) to $p$ (excluded).

Claim c. $G$ is $l$-c-free.

Suppose $L=\left\{e_{1}, \ldots, e_{l}\right\}$ is an $l$-c in $G^{*}$. Direct each edge $e_{i}$ in such a way that all other edges of $L$ lie on the left side of $e_{i}$. Denote by $S_{i}$ the half-open right side of $e_{i}$ as defined above. The $l$ sets $S_{i}$ are pairwise disjoint, and each one contains $\delta+1$ points of $A$. Contradiction to $\# A=m=(\delta+1) l-1$.

Claim d. $G$ is l-c-saturated.

Let $e^{*}$ be an edge of $\mathrm{CK}(n)$ not in $G$. Suppose that $e^{*}=[p, q]$, and the half-open arc $\left[p \rightarrow q\right.$ ) contains at most $\delta$ points of $A$. Let $a_{i}$ be the first point of $A$ encountered on bd $P$, starting at $q$ and going counterclockwise (possibly $a_{i}=q$ ). The only points of $A$ 
that may possibly lie in $\left[p \rightarrow q\right.$ ) are $a_{i+m-1}, a_{i+m-2}, \ldots, a_{i+m-\delta}$. Consider the edges

$$
e_{v}=\left[a_{i+v(\delta+1)},\left(a_{i+v(\delta+1)+\delta}\right)^{\prime}\right], \quad v=0,1, \ldots, l-2 .
$$

The second vertex of the last edge $e_{l-2}$ is $\left(a_{i+(l-2)(\delta+1)+\delta}\right)^{\prime}$. However,

$$
i+(l-2)(\delta+1)+\delta<i+m-\delta
$$

and therefore $e_{0}, e_{1}, \ldots, e_{l-2}, e^{*}$ is an $l-\mathrm{c}$ in $G \cup\left\{e^{*}\right\}$.

Next we show (for $l \geq 3, n \geq l$ ) that every $l$-c-saturated cgg is of the type described in (b) of Section 9.2. Here we follow the treatment in Section 4.

Let $G=(V, E)$ be an $l$-c-saturated $\operatorname{cgg}, \# V=n \geq l \geq 3$. Define $G^{*}=\left(V, E^{*}\right)=$ $\operatorname{Ext}(G)$, and put $m=\# E^{*}$. As in Section $4, m \geq 2$. Assume $E^{*}=\left\{e_{1}, \ldots, e_{m}\right\}$, $e_{i}=\left[a_{i}, b_{i}\right](i=1, \ldots, m)$, and no edge of $G$ (except $\left.e_{i}\right)$ lies on the right side of $e_{i}$, directed from $a_{i}$ to $b_{i}$. Define $A=\left\{a_{1}, \ldots, a_{m}\right\}, B=\left\{b_{1}, \ldots, b_{m}\right\}$ and note that $\# A=\# B=m$, as in Section 4 .

First we show that $B=A^{\prime}$. Since both sets are of the same size $m$, it suffices to show that $A^{\prime} \subset B$. Pick $i, 1 \leq i \leq m$. If $e_{i}$ is a boundary edge of $P(=[V])$, then $a_{i}^{\prime}=b_{i} \in B$. Otherwise, define $e_{i}^{\prime}=\left[a_{i}^{\prime}, b_{i}\right]$, and note that $e_{i}^{\prime} \notin E$. It follows that the set $E \cup\left\{e_{i}^{\prime}\right\}$ includes an $l$-c $L^{\prime}$, which must contain $e_{i}^{\prime}$. If $L^{\prime}$ also contains $e_{i}$, then $L^{\prime}$ cannot contain any other edge on $V$, except $\left[a_{i}, a_{i}^{\prime}\right]$. However, $\left[a_{i}, a_{i}^{\prime}\right]$ lies on the right side of $e_{i}$, and therefore is not in $E$. Since $l \geq 3$, this is impossible.

If $e_{i} \notin L^{\prime}$, then each edge of $L^{\prime}$ except $e_{i}^{\prime}$ can be replaced by an extreme edge of $G$ that lies "behind" it. (See Proposition 1.2 and Remark 1.5.) Assume therefore that $L^{\prime} \subset\left(E^{*} \backslash\left\{e_{i}\right\}\right) \cup\left\{e_{i}^{\prime}\right\}$. If no edge of $L^{\prime} \backslash\left\{e_{i}^{\prime}\right\}$ ends at $a_{i}^{\prime}$, then we can replace $e_{i}^{\prime}$ by $e_{i}$ and obtain an $l$-c in $G$, which is impossible. Thus $a_{i}^{\prime} \in B$.

By now we can write $e_{i}=\left[a_{i}, b_{i}\right]=\left[a_{i},\left(a_{i+\delta(i)}\right)^{\prime}\right]$, where $0 \leq \delta(i) \leq m-1$ and the function $\delta$ is $m$-periodic. If $\delta$ is not a constant, then, for some $i, \delta(i+1)<\delta(i)$, which implies that $e_{i+1}$ lies on the right side of $e_{i}$, a contradiction.

Thus $E^{*}=\left\{\left[a_{i},\left(a_{i+\delta}\right)^{\prime}\right]: i=1, \ldots, m\right\}$, for some constant $\delta, 0 \leq \delta<m$. It only remains to be shown that $m=(\delta+1) l-1$. ( $n \geq m$ is obvious.) If $m \geq(\delta+1) l$, then $\left\{e_{1+(\delta+1) \nu}: v=0,1, \ldots, l-1\right\}$ is an $l$-c in $E^{*}$. If $m<(\delta+1) l-1$, we distinguish two cases:

If $\delta=0$ and $m<(\delta+1) l-1=l-1$, then $E^{*}$ consists of at most $l-2$ boundary edges of $P$. Adding another boundary edge will not introduce an $l$-c (note that $n \geq l$ ).

If $\delta \geq 1$ and $m \leq(\delta+1) l-2$, define $e^{\prime}=\left[a_{1}, a_{1+\delta}\right] . e^{\prime} \notin E$, since it lies on the right side of $e_{1}$. The half-open $\operatorname{arc}\left[a_{1} \rightarrow a_{1+\delta}\right.$ ) on bd $P$ contains exactly $\delta$ points of $A$. Define $G^{\prime}=\left(V, E \cup\left\{e^{\prime}\right\}\right)$. If $L^{\prime}$ is an $l$-c in $G^{\prime}$, then either:

(1) $e^{\prime} \notin L^{\prime}$, i.e., $L^{\prime} \subset E$, which is impossible (see above, Claim c in Section 9.2).

(2) $e^{\prime} \in L^{\prime}$, but $e_{1} \notin L^{\prime}$. In that case we can replace each edge of $L^{\prime}$ by an extreme edge, to obtain an $l$-c $L \subset\left(E^{*} \backslash\left\{e_{1}\right\}\right) \cup\left\{e^{\prime}\right\}$. This is again impossible, by the same reasoning as in (1).

(3) $e^{\prime} \in L^{\prime}$ and $e_{1} \in L^{\prime}$. In this case $\# L^{\prime}=3$, and the third edge must be $\left[a_{1+\delta},\left(a_{1+\delta}\right)^{\prime}\right]$. However, this edge is not in $E$, since it lies on the right side of $e_{1}$. 
Proof of $(e)$ in Section 9.1. Assume $l \geq 3, n \geq l$, and let $G=(V, E)$ be an $l$-csaturated graph of order $n$. We relate the number of edges of $G$ to its structure, find the minimum, and determine the (unique) minimal cgg.

Let $P, \delta, m$, and $A=\left\{a_{1}, \ldots, a_{m}\right\}$, let $E^{*}$ and $G^{*}$ be defined as in (b) of Section 9.2, and assume $G=\operatorname{Bet}\left(G^{*}\right)$.

Two distinct vertices $p, q$ of $P$ divide the boundary of $P$ into two half-open arcs $[p \rightarrow q)$ and $[q \rightarrow p)$. The segment $[p, q]$ is an edge of $G$ iff each of these two arcs contains at least $\delta+1$ points of $A$. (See Claim b in Section 9.2.)

For $i=1, \ldots, m$ denote by $X_{i}$ the set of vertices of $P$ that lie strictly between $a_{i-1}$ and $a_{i}$, i.e., $X_{i}=V \cap\left(a_{i-1} \rightarrow a_{i}\right)$, where $a_{0}=a_{m}$. Define also $X_{i}^{+}=X_{i} \cup\left\{a_{i}\right\}$, and extend the definition of $X_{i}$ and $X_{i}^{+}$cyclically $(\bmod m)$ to all integers $i$.

From the above characterization of edges of $G$ we conclude that a vertex $x \in X_{i}^{+}$is not joined by an edge to any vertex in $X_{j}^{+}$, for $i-\delta \leq j \leq i+\delta$, and is joined to all other vertices of $G$. It follows that the number of edges $\left[a_{i}, a_{j}\right]$ is $\frac{1}{2} m(m-2 \delta-1)$, the number of edges $[x, a](x \in V \backslash A$ fixed, $a \in A)$ is $m-2 \delta-1$, and the total number of edges connecting $A$ with $V \backslash A$ is $(n-m)(m-2 \delta-1)$.

The number of edges connecting two vertices of $V \backslash A$ may be as low as zero. This happens (if and only if) all the nonempty sets $X_{i}$ lie within a short run of $\delta+1$ consecutive arcs: $\left\{X_{i}: i_{0} \leq i \leq i_{0}+\delta\right\}$. (Note that $n \geq m=(\delta+1) l-1 \geq 3 \delta+2$, and therefore $n-2 \delta>\delta$.)

Thus the minimum possible number of edges of $G$, for given $n, l$, and $\delta$, is

$$
\left(n-\frac{1}{2} m\right)(m-2 \delta-1)=\frac{1}{2}(2 n+1-(\delta+1) l)(\delta+1)(l-2) .
$$

This is a quadratic polynomial in $\delta+1$, strictly concave and symmetric about the axis $\delta+1=(2 n+1) / 2 l=\left(n+\frac{1}{2}\right) / l$. The effective domain of $\delta+1$ is $1 \leq \delta+1 \leq[(n+1) / l]$. As long as $n+1<2 l$, this is a single point.

In any case, the axis of symmetry $\left(n+\frac{1}{2}\right) / l$ lies strictly to the right of the center of the domain, $\frac{1}{2}(1+[(n+1) / l])$. (We urge the reader to verify this inequality.) It follows that the minimum of $(*)$ is attained when $\delta+1=1$, i.e., $\delta=0$, and only there.

The minimal $l$-c-saturated $\mathrm{cgg}$ of order $n$ is therefore unique, up to isomorhpism. It is obtained when $\delta=0, m=l-1$, and, say,

$$
A=\{v(0), v(1), \ldots, v(l-2)\}, \quad E^{*}=\{[v(i-1), v(i)]: i=1,2, \ldots, l-1\} .
$$

The vertices $v(1), \ldots, v(l-2)$ are universal, and $G$ is obtained from $\operatorname{CK}(n)$ by removing all edges on $v(l-1), v(l), \ldots, v(n-1), v(n)(=v(0))$. In particular, $c_{l}(n)=\left(\begin{array}{l}n \\ 2\end{array}\right)-$ $\left(\begin{array}{c}n-l+2 \\ 2\end{array}\right)$.

It is worth mentioning that the unique minimal $l$-c-saturated $\operatorname{cgg}$ of order $n$ described here is isomorphic, as an abstract graph, to the unique minimal $\mathbf{K}_{l}$-saturated graph of order $n$, as determined in [EHM]. However, we do not know of any simple way to deduce our result from that of [EHM], or vice versa. 


\section{Possible Extensions and Open Problems}

Consider a complete cgg CK $(n)$ based on a convex $n$-gon $P$. For $2 \leq l \leq n$ denote by $C_{l}$ the class of all convex sets of $l$ edges of $\mathrm{CK}(n)$.

A partial order $\leq$ can be defined on $C_{l}$, as follows:

$$
\left\{a_{1}, \ldots, a_{l}\right\} \leq\left\{b_{1}, \ldots, b_{l}\right\}
$$

if each $b_{i}$ lies (weakly) "beyond" $a_{i}$, i.e., if $b_{i}$ is weakly separated by the line aff $a_{i}$ from all other edges in $\left\{a_{1}, \ldots, a_{l}\right\}$.

Note that the class of all convex $l$-matchings in $\mathrm{CK}(n)$, as well as the class of all convex sets of $l$ edges in $\mathrm{CK}(n)$, form a "filter," i.e., an upwardly closed class, with respect to the partial order $\leq$.

There are many other natural classes of "forbidden configurations," which are filters with respect to $\leq$. To list just a few:

(A) For $\varepsilon=0,1, \ldots, l$ : convex sets of $l$ edges that use at least $l+\varepsilon$ vertices. For $1 \leq \varepsilon \leq l$, this is the same as convex sets of $l$ edges that form $\varepsilon$ or more disjoint simple paths. In this paper we have treated the cases $\varepsilon=l$ ( $l$-matchings) and $\varepsilon=0$ (no extra restrictions) extensively.

(B) For $\varepsilon=1, \ldots, l$ : convex sets of $l$ edges that have at least $\varepsilon$ odd components. (An odd component is a maximal path with an odd number of edges.)

(C) For $\alpha \geq 0$, convex $l$-matchings, with at least $\alpha$ extra vertices of $\operatorname{CK}(n)$ between any two edges.

For all these classes of forbidden configurations, the analog of Proposition 1.4 holds, i.e., the corresponding saturated cgg's satisfy $G=\operatorname{Bet}(\operatorname{Ext}(G))$.

In many cases an analog of Lemma 7.1 will hold: if all valences of $G$ exceed a certain natural lower bound, then $G$ must include a forbidden configuration.

It seems, however, that considerable extra effort will be needed to extend the extremal theories developed in this paper, and even to determine just the maximal functions, i.e., the analogs of $\mathrm{CM}_{l}(n)$ and $C_{l}(n)$.

\section{References}

[B] B. Bollobás, Graph Theory: An Introductory Course, Springer-Verlag, New York, 1979.

[E] P. Erdös, On sets of distances of $n$ points, Amer. Math. Monthly, 53 (1946), 248-250.

[EHM] P. Erdös, A. Hajnal, and J. Moon, A problem in graph theory, Amer. Math. Monthly, 71 (1964), 1107-1110.

[K] Y.S. Kupitz, Extremal Problems in Combinatorial Geometry, Aarhus University Lecture Notes Series, Vol. 53, 1979.

[T] P. Turán, Eine Extremalaufgabe aus der Graphentheorie, Mat.-Fiz. Lapok, 48 (1941), 436 -452.

[W] D. R. Woodall, Thrackles and deadlock, in: Combinatorial Mathematics and Its Applications (Proc. Conf., Oxford, 1969, ed. by D. J. A. Welsh), Academic Press, New York, 1971, pp. 335-347.

Received January 15, 1994, and in revised form July 15, 1995. 